\title{
Impact factor for high-energy two and three jets diffractive production
}

\author{
R. Boussarie, ${ }^{a}$ A.V. Grabovsky, ${ }^{b, c}$ L. Szymanowski ${ }^{d}$ and S. Wallon ${ }^{a, e}$ \\ ${ }^{a}$ Laboratoire de Physique Théorique, Bât. 210, Université Paris-Sud, CNRS, \\ 91405 Orsay, France \\ ${ }^{b}$ Physics Department, Novosibirsk State University, \\ 2 Pirogova street, Novosibirsk, Russia \\ ${ }^{c}$ Theory division, Budker Institute of Nuclear Physics, \\ 11 Lavrenteva avenue, Novosibirsk, Russia \\ ${ }^{d}$ Theoretical Physics Division, National Centre for Nuclear Research (NCBJ), \\ Hoza 69, 00-681 Warsaw, Poland \\ e UPMC Université Paris 06, Faculté de Physique, \\ 4 place Jussieu, 75252 Paris Cedex 05, France \\ E-mail: Renaud.Boussarie@th.u-psud.fr, A.V.Grabovsky@inp.nsk.su, \\ Lech.Szymanowski@fuw.edu.pl, Samuel.Wallon@th.u-psud.fr
}

ABSTRACT: We present the calculation of the impact factor for the $\gamma^{(*)} \rightarrow q \bar{q} g$ transition within Balitsky's high energy operator expansion. We also rederive the impact factor for the $\gamma^{(*)} \rightarrow q \bar{q}$ transition within the same framework. These results provide the necessary building blocks for further phenomenological studies of inclusive diffractive deep inelastic scattering, as well as, for two and three jets diffractive production, which go beyond approximations discussed in the literature.

KEYwords: Deep Inelastic Scattering, QCD

ARXIV EPRINT: 1405.7676 


\section{Contents}

1 Introduction 1

2 Definitions and necessary intermediate results 4

$\begin{array}{lll}3 & \text { LO impact factor for } \gamma \rightarrow q \bar{q} \text { transition } & 7\end{array}$

4 General expression for $\gamma \rightarrow q \bar{q} g$ impact factor $\quad 9$

5 Diagrams with the gluon crossing the shockwave 9

6 Diagrams without the gluon crossing the shockwave 13

$\begin{array}{lll}7 & \text { Impact factor for interaction with a color dipole } & 14\end{array}$

8 Impact factor in the momentum space and in the linear approximation 18

9 Conclusions $\quad 20$

$\begin{array}{ll}\text { A Integrals necessary for linearization } & 21\end{array}$

$\begin{array}{ll}\text { B Integrals necessary for Fourier transform } & 23\end{array}$

\section{Introduction}

Diffraction is one of the key tools to understand the dynamics of strong interaction, and it has been studied since the sixties. In particular, the research program performed at HERA has shown that semi-hard diffractive processes, in which a hard scale allows one to deal with QCD in its perturbative regime, could provide a quantitative lever-arm to understand the internal dynamics of the nucleon in a regime of very high gluon densities. ${ }^{1}$ Among the whole set of $\gamma^{*} p \rightarrow X$ deep inelastic scattering (DIS) events, almost $10 \%$ reveal a rapidity gap between the proton remnants and the hadrons coming from the fragmentation region of the initial virtual photon, so that the process looks rather like $\gamma^{*} p \rightarrow X Y$ [3-10], where $Y$ is the outgoing proton or one of its low-mass excited states. This subset of events is called diffractive deep inelastic scattering (DDIS). DDIS can be studied at the inclusive level, or further analyzed by considering diffractive jet production as well as exclusive meson production. One of the main cornerstones of diffraction is the concept of Pomeron, which carries the quantum numbers of vacuum and which is exchanged at high energy between $X$ and $Y$. Diffraction can be described according to

\footnotetext{
${ }^{1}$ For reviews, see refs. [1, 2].
} 
several approaches, important for phenomenological applications. In the perturbative QCD approach, justified by the existence of a hard scale (like the photon virtuality $Q^{2}$ ), one can rely on a QCD factorization theorem [11]. This is the essence of the first approach, which involves a resolved Pomeron contribution: the diffractive structure function is expressed as the convolution of a coefficient function with a diffractive parton distribution, which is analogous to the usual parton distribution function (PDF), but with the proton replaced by a Pomeron.

Besides, at high energies, it is natural to model the diffractive events by a direct Pomeron contribution involving the coupling of a Pomeron with the diffractive state. The diffractive states can be modelled in perturbation theory by a $q \bar{q}$ pair (for moderate $M^{2}$, where $M$ is the invariant mass of the diffractively produced state $X$ ) or by higher Fock states as a $q \bar{q} g$ state for larger values of $M^{2}$. Based on such a model, with a two-gluon exchange picture for the Pomeron, a good description of HERA data for diffraction could be achieved [12]. One of the important features of this approach is that the $q \bar{q}$ component with a longitudinally polarized photon plays a crucial role in the region of small diffractive mass $M$, although it is a twist- 4 contribution. A further analysis was then performed, combining both the resolved and the direct components $[13,14]$, including a $q \bar{q}$ exchange on top of the gluon pair to model the Pomeron.

In the direct components considered there, the $q \bar{q} g$ diffractive state has been studied in two particular limits. The first one, valid for very large $Q^{2}$, corresponds to a collinear approximation in which the transverse momentum of the gluon is assumed to be much smaller than the transverse momentum of the emitter $[15,16]$. This approximation allows one to extract the leading logarithm in $Q^{2}$, based on the strong ordering of transverse momenta typical of DGLAP evolution [17-20]. The second one [21, 22], valid for very large $M^{2}$, is based on the assumption of a strong ordering of longitudinal momenta, encountered in BFKL equation [23-26]. A model combining both these limits was elaborated and applied to HERA data for DDIS in ref. [27].

The main aim of the present article is to compute the $\gamma^{*} \rightarrow q \bar{q} g$ impact factor and to rederive the $\gamma^{*} \rightarrow q \bar{q}$ impact factor, both at tree level, with an arbitrary number of $t$-channel gluons, here described within the Wilson line formalism, also called QCD shockwave approach [28-31]. In particular, the $\gamma^{*} \rightarrow q \bar{q} g$ transition is computed without any soft or collinear approximation for the emitted gluon, in contrast with the above mentioned calculations. These results provide necessary generalization of buiding blocks for inclusive DDIS as well as for two- and three-jet diffractive production, in the fragmentation region of the scattered photon, i.e. in the forward rapidity region. Since the results we derived can account for an arbitrary number of $t$-channel gluons, this could allow one to include higher twist effects which are suspected to be rather important in DDIS for $Q^{2} \lesssim 5 \mathrm{GeV}^{2}$ [32].

The QCD shock-wave approach on which we rely is an operator language based on the concept of factorization of the scattering amplitude in rapidity space and on the extension to high-energy (Regge limit) of the Operator Product Expansion (OPE) technique, which was only known at moderate energy (Bjorken limit) before, as an expansion in terms of local operators or in terms of light-ray operators [33]. In DIS off a hadron at high-energy, the matrix elements made of Wilson line operators appearing in the OPE describe the 
non perturbative part of the process, and their evolution in rapidity is related to the evolution of the structure function of the target. The evolution equation can be obtained relying on background field techniques. The Wilson-line operators in the high-energy OPE evolve with respect to rapidity according to the Balitsky equation, which reduces to the Balitsky-Kovchegov (BK) equation $[28-31,34,35]$ in the large $N_{c}$ limit. According to the best of our knowledge, this shock-wave approach was only used for evolution equations and for impact factors at inclusive level for the $\gamma^{*} \rightarrow \gamma^{*}$ impact factor at next-to-leading order [36, 37], and at semi-inclusive level for $p_{t}$-broadening in $p-A$ collisions in refs. [38, 39]. Its application shows that this method is very powerful [40] when compared with usual methods based on summation of contributions of individual Feynman diagrams computed in momentum space.

When describing a diffractive process, the above mentioned approach is natural in order to implement saturation effects at high energies, since it is formulated in the coordinate space. Indeed, in the dipole picture [41, 42], when probing a nucleon with a virtual photon in the rest frame of the nucleon, due to the long life-time of the virtual $q \bar{q}$ pair produced by the $\gamma^{*}$ probe with respect to its scattering time, this pair is almost frozen during its interaction. The inclusive cross-section (for DIS) as well as the scattering amplitude (for DDIS) thus naturally factorizes in the coordinate space in terms of an impact factor involving a dipole of given transverse size $r$ convoluted with an effective dipole-nucleon cross-section $\sigma(x, r)$, a function of Bjorken $x$ and $r$. The same picture was extended to the $q \bar{q} g$ intermediate state, at least in the collinear approximation in which case this intermediate state can be considered as a gluon-gluon dipole $[15,16]$, the $q \bar{q}$ being an effective gluon due to its localization in the transverse coordinate space (since the relative transverse momentum of this pair is large with respect to the transverse momentum of the emitted gluon). A step further in this spirit was done in the case of vector meson electroproduction at twist 3 level, including the genuine twist 3 contribution which involves a $q \bar{q} g$ intermediate state [43, 44], for which a dipole picture was also obtained [45], based on $\mathrm{QCD}$ equations of motion.

This dipole picture provides the natural framework for the formulation of saturation. Indeed, the transverse size $r$ of the dipole is the natural parameter in order to implement both color transparency (for small $r$ ) and saturation (for large $r$ ). The analysis of low- $x$ saturation dynamics of the nucleon target was first introduced in refs. $[46,47]$ by Golec-Biernat and Wüsthoff (GBW) to describe the inclusive and diffractive structure functions of DIS. This is an additional reason to rely on the shock-wave analysis, which naturaly provides a tool to evaluate the $\gamma^{*} \rightarrow q \bar{q}$ and $\gamma^{*} \rightarrow q \bar{q} g$ impact factors in transverse coordinate space.

The paper is organized as follows. The section 2 contains the definitions and necessary intermediate results. Section 3 briefly reproduces the leading order (LO) $\gamma^{*} \rightarrow q \bar{q}$ impact factor. In section 4 we give the general expression for the $\gamma \rightarrow q \bar{q} g$ impact factor, which is then calculated in sections 5 and 6 . Section 7 discusses the linearized impact factor for interaction with the color dipole. Section 8 is devoted to the impact factor in the momentum space. Section 9 summarizes obtained results. Two appendices comprise necessary technical details. 


\section{Definitions and necessary intermediate results}

Throughout this paper, we use the following notations. We introduce the light cone vectors $n_{1}$ and $n_{2}$

$$
n_{1}=(1,0,0,1), \quad n_{2}=\frac{1}{2}(1,0,0,-1), \quad n_{1}^{+}=n_{2}^{-}=n_{1} \cdot n_{2}=1,
$$

and for any vector $p$ we have

$$
\begin{array}{rlrl}
p^{+} & =p_{-}=p \cdot n_{2}=\frac{1}{2}\left(p^{0}+p^{3}\right), & & p_{+}=p^{-}=p \cdot n_{1}=p^{0}-p^{3}, \\
p & =p^{+} n_{1}+p^{-} n_{2}+p_{\perp}, & & p^{2}=2 p^{+} p^{-}-\vec{p}^{2}, \\
p \cdot k & =p^{\mu} k_{\mu}=p^{+} k^{-}+p^{-} k^{+}-\vec{p} \cdot \vec{k}= & p_{+} k_{-}+p_{-} k_{+}-\vec{p} \cdot \vec{k} .
\end{array}
$$

The derivatives and the metric tensor have the form

$$
\begin{aligned}
\partial_{ \pm} & =\partial^{\mp}=\frac{\partial}{\partial z^{ \pm}}=\frac{\partial}{\partial z_{\mp}}, \quad \partial_{i}=-\partial^{i}=\frac{\partial}{\partial z^{i}}=-\frac{\partial}{\partial z_{i}}, \\
g^{\mu \nu} & =g_{\mu \nu}=\left(\begin{array}{rrrr}
0 & 1 & 0 & 0 \\
1 & 0 & 0 & 0 \\
0 & 0 & -1 & 0 \\
0 & 0 & 0 & -1
\end{array}\right), \quad \text { the indices are }+,-, 1,2 ; \\
\epsilon^{+\beta \gamma-} & =-\epsilon^{0 \beta \gamma 3}=-e^{\beta \gamma 3} .
\end{aligned}
$$

We denote the initial photon momentum as $k$, and the outgoing quark, antiquark, and gluon momenta as $p_{q}, p_{\bar{q}}$, and $p_{g}$. The corresponding longitudinal momentum fractions are

$$
\frac{p_{q}^{+}}{k^{+}}=x_{q}, \quad \frac{p_{\bar{q}}^{+}}{k^{+}}=x_{\bar{q}}, \quad \frac{p_{g}^{+}}{k^{+}}=x_{g} .
$$

For simplicity, we work with a photon in the forward kinematics

$$
\vec{k}=0, \quad k^{\mu}=k^{+} n_{1}^{\mu}+\frac{k^{2}}{2 k^{+}} n_{2}^{\mu}, \quad-k^{2}=Q^{2}>0 .
$$

Its longitudinal and transverse polarization vectors read

$$
\begin{aligned}
\varepsilon_{L}^{\alpha} & =\frac{1}{\sqrt{-k^{2}}}\left(k^{+} n_{1}^{\alpha}-\frac{k^{2}}{2 k^{+}} n_{2}^{\alpha}\right), & \varepsilon_{L}^{+} & =\frac{k^{+}}{Q}, \quad \varepsilon_{L}^{-}=\frac{Q}{2 k^{+}}, \\
\varepsilon_{T}^{\alpha} & =\varepsilon_{T \perp}^{\alpha}=\frac{1}{\sqrt{2}}(0,1, i s, 0), & s & = \pm 1 .
\end{aligned}
$$

Here $s$ is the helicity of the photon. For the outgoing gluon we work in the light cone gauge $A \cdot n_{2}=0$. Therefore

$$
\varepsilon_{g \nu}^{*}=\frac{\left(\vec{\varepsilon}_{g}^{*} \cdot \vec{p}_{g}\right)}{p_{g}^{+}} n_{2 \nu}+\varepsilon_{g \perp \nu}^{*}=\left(g_{\perp \nu \alpha}-\frac{p_{g \perp \alpha} n_{2 \nu}}{p_{g}^{+}}\right) \varepsilon_{g}^{* \alpha},
$$

and we can use the same transverse polarization vectors as for the photon

$$
\varepsilon_{g \perp}^{* \alpha}=\frac{1}{\sqrt{2}}\left(0,1,-i s_{g}, 0\right), \quad s_{g}= \pm 1 .
$$


It is convenient to introduce the following vectors

$$
\vec{P}_{\bar{q}}=\frac{\vec{p}_{g}}{x_{g}}-\frac{\vec{p}_{\bar{q}}}{x_{\bar{q}}}, \quad \vec{P}_{q}=\frac{\vec{p}_{g}}{x_{g}}-\frac{\vec{p}_{q}}{x_{q}}
$$

Then

$$
\left(\vec{P}_{\bar{q}} \cdot \vec{\varepsilon}_{g}^{*}\right)=\frac{p_{\bar{q}} \cdot \varepsilon_{g}^{*}}{x_{\bar{q}}}, \quad\left(\vec{P}_{q} \cdot \vec{\varepsilon}_{g}^{*}\right)=\frac{p_{q} \cdot \varepsilon_{g}^{*}}{x_{q}} .
$$

To simplify the vector products with the polarization vectors we will use the following identities

$$
\left[\vec{a} \times \vec{\varepsilon}_{T}\right]=i s\left(\vec{a} \cdot \vec{\varepsilon}_{T}\right), \quad\left[\vec{a} \times \vec{\varepsilon}_{g}^{*}\right]=-i s_{g}\left(\vec{a} \cdot \vec{\varepsilon}_{g}^{*}\right),
$$

where $[\vec{a} \times \vec{b}] \equiv e^{\gamma \beta 3} a^{\gamma} b^{\beta}$. The fermion propagator in the shock wave background can be read from ref. [28] and is given by

$$
\begin{aligned}
G\left(z_{1}, z_{2}\right)= & \theta\left(z_{1}^{+} z_{2}^{+}\right) G_{0}\left(z_{12}\right)-\int d^{4} z_{3} \delta\left(z_{3}^{+}\right) G_{0}\left(z_{13}\right) \gamma^{+} G_{0}\left(z_{32}\right) \\
& \times\left(\theta\left(z_{1}^{+}\right) \theta\left(-z_{2}^{+}\right) U_{\vec{z}_{3}}+\theta\left(-z_{1}^{+}\right) \theta\left(z_{2}^{+}\right) U_{\vec{z}_{3}}^{\dagger}\right) .
\end{aligned}
$$

Here $z_{i j}=z_{i}-z_{j}$. The free quark propagator reads

$$
G_{0}(x)=\frac{2 i}{(2 \pi)^{2}} \frac{\hat{z}}{\left(z^{2}-i 0\right)^{2}}, \quad G_{0}(p)=\frac{i \hat{p}}{p^{2}+i 0},
$$

and the Wilson lines

$$
U_{i}=U_{\vec{z}_{i}}=U\left(\vec{z}_{i}, \eta\right)=P \exp \left[i g \int_{-\infty}^{+\infty} b_{\eta}^{-}\left(z_{i}^{+}, \vec{z}_{i}\right) d z_{i}^{+}\right]
$$

are integrated along the path $z^{-}=0$. The operator $b_{\eta}^{-}$is the external shock-wave field built from only slow gluons which momenta are limited by the longitudinal cut-off defined by the rapidity $\eta$

$$
b_{\eta}^{-}=\int \frac{d^{4} p}{(2 \pi)^{4}} e^{-i p \cdot z} b^{-}(p) \theta\left(e^{\eta}-\left|p^{+}\right|\right) .
$$

We use the light cone gauge

$$
\mathcal{A} \cdot n_{2}=0,
$$

with $\mathcal{A}$ being the sum of the external field $b$ and the quantum field $A$

$$
\mathcal{A}=A+b, \quad b^{\mu}(z)=b^{-}\left(z^{+}, \vec{z}\right) n_{2}^{\mu}=\delta\left(z^{+}\right) B(\vec{z}) n_{2}^{\mu} .
$$

Using the LSZ reduction formulas for the propagators from ref. [28] or summing the diagrams in this external shockwave field as in ref. [28] one can get the external fermion lines in the shockwave background. In the following calculations, we will only need those external lines for $y^{+}<0$, which read

$$
\begin{aligned}
& \left.\bar{u}(p, y)\right|_{0>y^{+}}=\int d^{4} z \delta\left(z^{+}\right) e^{i p \cdot z} \frac{\bar{u}_{p}}{\sqrt{2 p^{+}}} \gamma^{+} U_{\vec{z}} G_{0}(z-y), \\
& \left.v(p, y)\right|_{0>y^{+}}=-\int d^{4} z \delta\left(z^{+}\right) e^{i p \cdot z} G_{0}(y-z) U_{\vec{z}}^{\dagger} \gamma^{+} \frac{v_{p}}{\sqrt{2 p^{+}}} .
\end{aligned}
$$


In the same way one can get the gluon external line in the shockwave background

$$
\left.\epsilon_{\nu}^{*}(p, y)\right|_{0>y^{+}}=-4 p^{+} \varepsilon^{* \alpha} \theta\left(p^{+}\right) \int \frac{d^{4} z \delta\left(z^{+}\right)}{(2 \pi)^{2}} \frac{e^{i p \cdot z}}{\sqrt{2 p^{+}}} U_{\vec{z}} \frac{1}{\frac{\partial}{\partial y^{-}}} \frac{g_{\perp \alpha \nu}\left(-y^{+}\right)-(z-y)_{\perp \alpha} n_{2 \nu}}{\left((z-y)^{2}-i 0\right)^{2}}
$$

where we have introduced the notation

$$
\frac{1}{\frac{\partial}{\partial y}} f(y)=\int \frac{d p}{2 \pi} \int d u \frac{e^{-i p(y-u)}}{-i p} f(u)
$$

In eqs. (2.23), (2.24), the Wilson line is in the adjoint representation. If $U \rightarrow 1$, then

$$
\begin{aligned}
\left.\bar{u}(p, y)\right|_{0>y^{+}} & \rightarrow \theta\left(p^{+}\right) \frac{\bar{u}_{p}}{\sqrt{2 p^{+}}} e^{i p \cdot y},\left.\quad v(p, y)\right|_{0>y^{+}} \rightarrow \theta\left(p^{+}\right) \frac{v_{p}}{\sqrt{2 p^{+}}} e^{i p \cdot y}, \\
\left.\epsilon_{\nu}^{*}(p, y)\right|_{0>y^{+}} & \rightarrow \frac{\theta\left(p^{+}\right) \varepsilon_{p}^{* \alpha}}{\sqrt{2 p^{+}}}\left(g_{\perp \alpha \nu}-\frac{p_{\perp \alpha} n_{2 \nu}}{p^{+}}\right) e^{i p \cdot y}=\theta\left(p^{+}\right) \frac{\varepsilon_{p \nu}^{*}}{\sqrt{2 p^{+}}} e^{i p \cdot y},
\end{aligned}
$$

and we recover the results without the shockwave.

Below, we will need the following integral ${ }^{2}$ derived in ref. [37]

$$
\begin{aligned}
\int d^{4} z \frac{\hat{x}-\hat{z}}{(x-z)^{4}}\left[\gamma^{\mu} \frac{z^{\nu}}{z^{4}}-\gamma^{\nu} \frac{z^{\mu}}{z^{4}}\right] \frac{\hat{z}-\hat{y}}{(z-y)^{4}}= & \frac{-i \pi^{2}}{x^{2} y^{2}(x-y)^{2}}\left(\frac{\hat{x} \gamma^{\nu} \hat{y} x^{\mu}-\hat{x} \gamma^{\mu} \hat{y} x^{\nu}}{x^{2}}\right. \\
& +\frac{\hat{x} \gamma^{\nu} \hat{y} y^{\mu}-\hat{x} \gamma^{\mu} \hat{y} y^{\nu}}{y^{2}}+\frac{\left(\gamma^{\nu} \gamma^{\mu}-\gamma^{\mu} \gamma^{\nu}\right) \hat{y}}{2} \\
& \left.+\frac{\hat{x}\left(\gamma^{\mu} \gamma^{\nu}-\gamma^{\nu} \gamma^{\mu}\right)}{2}+2 \frac{y^{\mu} x^{\nu}-y^{\nu} x^{\mu}}{(x-y)^{2}}[\hat{y}-\hat{x}]\right),
\end{aligned}
$$

and the following representation of the McDonald functions

$$
-2 K_{0}(2 \sqrt{a b})=\int_{-\infty}^{0} \frac{d z}{z} e^{i(a-i 0) z-i \frac{b+i 0}{z}}, \quad K_{1}(r)=-K_{0}^{\prime}(r),
$$

together with the fact that they obey the Bessel equation

$$
\Delta K_{0}(r)=\left(\frac{\partial^{2}}{\partial r^{2}}+\frac{1}{r} \frac{\partial}{\partial r}\right) K_{0}(r)=K_{0}(r)+2 \pi \delta(\vec{r}) .
$$

We will also need the following Dirac structures

$$
\begin{aligned}
\bar{u}_{p_{q}} \gamma^{+} v_{p_{\bar{q}}} & =\lambda_{q} \bar{u}_{p_{q}} \gamma^{+} \gamma^{5} v_{p_{\bar{q}}}=\delta_{\lambda_{q},-\lambda_{\bar{q}}} \sqrt{2 p_{q}^{+} 2 p_{\bar{q}}^{+}}, \quad \lambda_{q}= \pm 1 \\
\bar{u}_{p_{q}} \gamma^{j} v_{p_{\bar{q}}} & =\lambda_{q} \bar{u}_{p_{q}} \gamma^{j} \gamma^{5} v_{p_{\bar{q}}}=\delta_{\lambda_{q},-\lambda_{\bar{q}}} \sqrt{2 p_{\bar{q}}^{+} 2 p_{q}^{+}}\left(\frac{p_{q}^{j}}{2 p_{q}^{+}}+\frac{p_{\bar{q}}^{j}}{2 p_{\bar{q}}^{+}}+i \varepsilon^{t j 3} \lambda_{q}\left(\frac{p_{q}^{t}}{2 p_{q}^{+}}-\frac{p_{\bar{q}}^{t}}{2 p_{\bar{q}}^{+}}\right)\right)
\end{aligned}
$$

where $\lambda_{q}$ is the quark helicity.

\footnotetext{
${ }^{2}$ The factor $-i$ in eq. (2.29) corrects a misprint from ref. [37].
} 


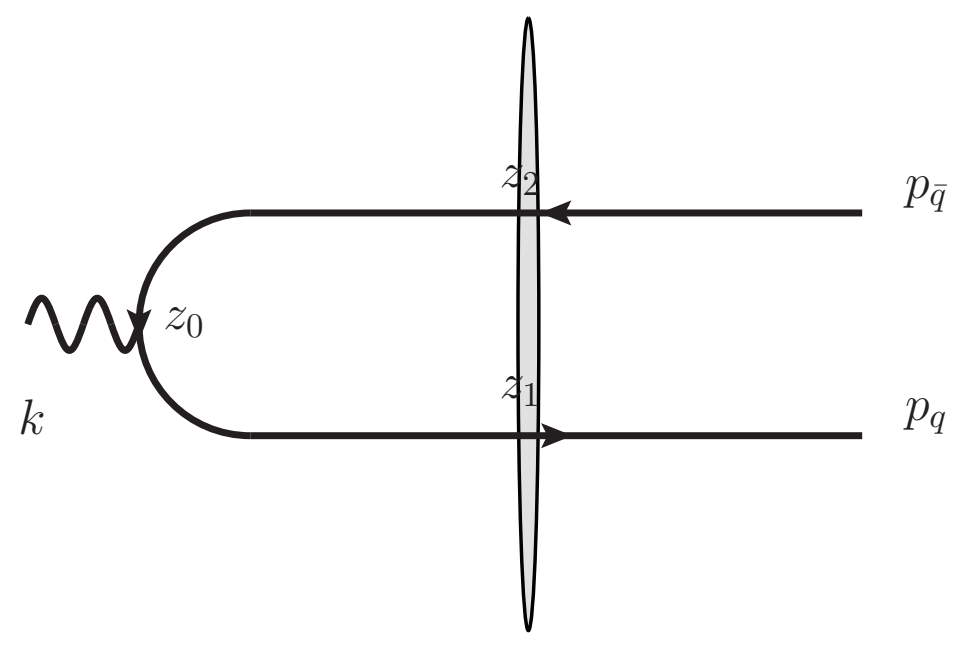

Figure 1. LO $\gamma \rightarrow q \bar{q}$ impact factor.

\section{LO impact factor for $\gamma \rightarrow q \bar{q}$ transition}

In this section we will briefly reproduce the known expression for the LO $\gamma^{*} \rightarrow q \bar{q}$ impact factor, see figure 1 , for completeness of this paper. The LO matrix element for the electromagnetic current in the shockwave background reads

$\tilde{M}_{0}^{\alpha}=\delta_{l}^{n} \frac{\left\langle 0\left|b_{p_{\bar{q}}}^{l}\left(a_{p_{q}}\right)_{n} \bar{\psi}\left(z_{0}\right) \gamma^{\alpha} \psi\left(z_{0}\right) e^{i \int \mathcal{L}_{i}(z) d z}\right| 0\right\rangle}{\left\langle 0\left|e^{i \int \mathcal{L}(z) d z}\right| 0\right\rangle}=\int d \vec{z}_{1} d \vec{z}_{2} F\left(p_{q}, p_{\bar{q}}, z_{0}, \vec{z}_{1}, \vec{z}_{2}\right)^{\alpha} \operatorname{tr}\left(U_{1} U_{2}^{\dagger}\right)$.

Here $a$ and $b$ are the quark and antiquark annihilation operators carrying colour indices $n$ and $l$,

$$
\begin{aligned}
F\left(p_{q}, p_{\bar{q}}, z_{0}, \vec{z}_{1}, \vec{z}_{2}\right)^{\alpha}= & \int d z_{1}^{-} d z_{1}^{+} \delta\left(z_{1}^{+}\right) \int d z_{2}^{-} d z_{2}^{+} \delta\left(z_{2}^{+}\right) \frac{e^{i p_{q} \cdot z_{1}+i p_{\bar{q}} \cdot z_{2}}}{\sqrt{2 p_{q}^{+} 2 p_{\bar{q}}^{+}}} \\
& \times \bar{u}_{p_{q}} \gamma^{+} G_{0}\left(z_{10}\right) \gamma^{\alpha} G_{0}\left(z_{02}\right) \gamma^{+} v_{p_{\bar{q}}} \\
= & \frac{\theta\left(p_{q}^{+}\right) \theta\left(p_{\bar{q}}^{+}\right) e^{-i \vec{p}_{q} \cdot \vec{z}_{1}-i \vec{p}_{\bar{q}} \cdot \vec{z}_{2}}}{4(2 \pi)^{4}\left(z_{0}^{+}\right)^{2} \sqrt{2 p_{q}^{+} 2 p_{\bar{q}}^{+}}} \\
& \times \bar{u}_{p_{q}} \gamma^{+}\left(i p_{q}^{+} \gamma^{-}-\gamma_{\perp}^{\beta} \frac{\partial}{\partial z_{1 \perp}^{\beta}}\right) \gamma^{\alpha}\left(i p_{\bar{q}}^{+} \gamma^{-}-\gamma_{\perp}^{\gamma} \frac{\partial}{\partial z_{2 \perp}^{\gamma}}\right) \gamma^{+} v_{p_{\bar{q}}} \\
& \exp \left[i p_{q}^{+}\left(z_{0}^{-}+\frac{-z_{10 \perp}^{2}+i 0}{-2 z_{0}^{+}}\right)\right] \exp \left[i p_{\bar{q}}^{+}\left(z_{0}^{-}+\frac{-z_{20 \perp}^{2}+i 0}{-2 z_{0}^{+}}\right)\right]
\end{aligned}
$$

We now introduce $M_{0}^{\alpha}$, built from $\tilde{M}_{0}^{\alpha}$ by substracting the non-interacting term, i.e.

$$
M_{0}^{\alpha}=\int d \vec{z}_{1} d \vec{z}_{2} F\left(p_{q}, p_{\bar{q}}, z_{0}, \vec{z}_{1}, \vec{z}_{2}\right)^{\alpha}\left(\operatorname{tr}\left(U_{1} U_{2}^{\dagger}\right)-N_{c}\right)
$$


The Fourier transform of $F$ w.r.t. $z_{0}$ is defined as

$$
F\left(p_{q}, p_{\bar{q}}, k, \vec{z}_{1}, \vec{z}_{2}\right)^{\alpha}=\int d^{4} z_{0} e^{-i k \cdot z_{0}} F\left(p_{q}, p_{\bar{q}}, z_{0}, \vec{z}_{1}, \vec{z}_{2}\right)^{\alpha}
$$

In the kinematics (2.9) we chose for the photon, we have

$$
\begin{aligned}
F\left(p_{q}, p_{\bar{q}}, k, \vec{z}_{1}, \vec{z}_{2}\right)^{\alpha}= & \theta\left(p_{q}^{+}\right) \theta\left(p_{\bar{q}}^{+}\right) \frac{i \delta\left(k^{+}-p_{q}^{+}-p_{\bar{q}}^{+}\right)}{2 k^{+}(2 \pi)^{2} \sqrt{2 p_{q}^{+} 2 p_{\bar{q}}^{+}}} e^{-i \vec{p}_{q} \cdot \vec{z}_{1}-i \vec{p}_{\bar{q}} \cdot \vec{z}_{2}} \\
& \times \bar{u}_{p_{q}} \gamma^{+}\left(i p_{q}^{+} \gamma^{-}-\gamma_{\perp}^{\beta} \frac{\partial}{\partial z_{1 \perp}^{\beta}}\right) \gamma^{\alpha}\left(i p_{\bar{q}}^{+} \gamma^{-}-\gamma_{\perp}^{\gamma} \frac{\partial}{\partial z_{2 \perp}^{\gamma}}\right) \gamma^{+} v_{p_{\bar{q}}} K_{0}\left(Q \sqrt{x_{q} x_{\bar{q}} \vec{z}_{12}^{2}}\right)
\end{aligned}
$$

Calculating the derivatives with $\alpha=-$ we will encounter

$$
\begin{aligned}
g_{\perp}^{\gamma \beta} \frac{\partial}{\partial z_{2 \perp}^{\gamma}} \frac{\partial}{\partial z_{1 \perp}^{\beta}} K_{0}\left(Q \sqrt{x_{q} x_{\bar{q}} \vec{z}_{12}^{2}}\right) & =\Delta_{\vec{z}_{12}} K_{0}\left(Q \sqrt{x_{q} x_{\bar{q}} \vec{z}_{12}^{2}}\right) \\
& =Q^{2} x_{q} x_{\bar{q}} K_{0}\left(Q \sqrt{x_{q} x_{\bar{q}} \vec{z}_{12}^{2}}\right)+2 \pi \delta\left(\vec{z}_{12}\right)
\end{aligned}
$$

where we used (2.31) to derive this expression. However, the term with the $\delta$ distribution will give no contribution to (3.3), therefore we can drop it. That being done, we get

$$
\begin{aligned}
F\left(p_{q}, p_{\bar{q}}, k, \vec{z}_{1}, \vec{z}_{2}\right)^{\alpha} \varepsilon_{L \alpha}= & \theta\left(p_{q}^{+}\right) \theta\left(p_{\bar{q}}^{+}\right) \frac{\delta\left(k^{+}-p_{q}^{+}-p_{\bar{q}}^{+}\right)}{(2 \pi)^{2}} e^{-i \vec{p}_{q} \cdot \vec{z}_{1}-i \vec{p}_{\bar{q}} \cdot \vec{z}_{2}} \\
& \times(-2 i) \delta_{\lambda_{q},-\lambda_{\bar{q}}} x_{q} x_{\bar{q}} Q K_{0}\left(Q \sqrt{x_{q} x_{\bar{q}} \vec{z}_{12}^{2}}\right)
\end{aligned}
$$

and

$$
\begin{aligned}
F\left(p_{q}, p_{\bar{q}}, k, \vec{z}_{1}, \vec{z}_{2}\right)^{j} \varepsilon_{T j}= & \theta\left(p_{q}^{+}\right) \theta\left(p_{\bar{q}}^{+}\right) \frac{\delta\left(k^{+}-p_{q}^{+}-p_{\bar{q}}^{+}\right)}{(2 \pi)^{2}} e^{-i \vec{p}_{q} \cdot \vec{z}_{1}-i \vec{p}_{\bar{q}} \cdot \vec{z}_{2}} \\
& \times \delta_{\lambda_{q},-\lambda_{\bar{q}}}\left(x_{q}-x_{\bar{q}}+s \lambda_{q}\right) \frac{\vec{z}_{12} \cdot \vec{\varepsilon}_{T}}{\vec{z}_{12}^{2}} Q \sqrt{x_{q} x_{\bar{q}} \vec{z}_{12}^{2}} K_{1}\left(Q \sqrt{x_{q} x_{\bar{q}} \vec{z}_{12}^{2}}\right) .
\end{aligned}
$$

Using the identity

$$
F^{+}=F^{-} \frac{2\left(k^{+}\right)^{2}}{Q^{2}}
$$

one can easily check that the electromagnetic gauge invariance

$$
F\left(p_{q}, p_{\bar{q}}, k, \vec{z}_{1}, \vec{z}_{2}\right)^{\alpha} k_{\alpha}=0
$$

is satisfied. The results (3.7) and (3.8) are consistent with the well known result for the $\gamma \rightarrow q \bar{q}$ wave-function which was derived for example in ref. [48]. 


\section{General expression for $\gamma \rightarrow q \bar{q} g$ impact factor}

We will extract the impact factor from the following matrix element

$$
\begin{aligned}
\tilde{M}^{\alpha}= & \left(t^{b}\right)_{l}^{n} \frac{\left\langle 0\left|c_{p_{g}}^{b} b_{p_{\bar{q}}}^{l}\left(a_{p_{q}}\right)_{n} \bar{\psi}\left(z_{0}\right) \gamma^{\alpha} \psi\left(z_{0}\right) e^{i \int \mathcal{L}_{i}(z) d z}\right| 0\right\rangle}{\langle 0| e^{i \int \mathcal{L}(z) d z|0\rangle}} \\
= & \int d \overrightarrow{z_{1}} d \vec{z}_{2} d \vec{z}_{3} F_{1}\left(p_{q}, p_{\bar{q}}, p_{g}, z_{0}, \vec{z}_{1}, \vec{z}_{2}, \vec{z}_{3}\right)^{\alpha} \operatorname{tr}\left(U_{1} t^{a} U_{2}^{\dagger} t^{b}\right) U_{3}^{b a} \\
& +\int d \overrightarrow{z_{1}} d \vec{z}_{2} \tilde{F}_{2}\left(p_{q}, p_{\bar{q}}, p_{g}, z_{0}, \vec{z}_{1}, \vec{z}_{2}\right)^{\alpha} \frac{N_{c}^{2}-1}{2 N_{c}} \operatorname{tr}\left(U_{1} U_{2}^{\dagger}\right) .
\end{aligned}
$$

Here $\left(t^{b}\right)_{l}^{k}$ is the projector to the color singlet state, and $c, a$, and $b$ are the gluon, quark and antiquark annihilation operators; $F_{1}$ describes the contribution of the first two diagrams and $\tilde{F}_{2}$ stands for diagrams 3 and 4 . The space coordinates $z_{0,1,2,3,4}$ and the momenta $p_{q, \bar{q}, g}$ are defined in figure 2 .

In this form $\tilde{M}$ contains contributions from terms without interaction in which all operators $U$ are reduced to identity. To get the impact factor we have to subtract those terms. This amounts in replacing $\tilde{M}$ by $M$, which reads

$$
\begin{aligned}
M^{\alpha}= & \int d \vec{z}_{1} d \vec{z}_{2} d \vec{z}_{3} F_{1}\left(p_{q}, p_{\bar{q}}, p_{g}, z_{0}, \vec{z}_{1}, \vec{z}_{2}, \vec{z}_{3}\right)^{\alpha}\left[\operatorname{tr}\left(U_{1} t^{a} U_{2}^{\dagger} t^{b}\right) U_{3}^{b a}-\frac{N_{c}^{2}-1}{2}\right] \\
& +\int d \vec{z}_{1} d \vec{z}_{2} \tilde{F}_{2}\left(p_{q}, p_{\bar{q}}, p_{g}, z_{0}, \vec{z}_{1}, \vec{z}_{2}\right)^{\alpha} \frac{N_{c}^{2}-1}{2 N_{c}}\left(\operatorname{tr}\left(U_{1} U_{2}^{\dagger}\right)-N_{c}\right) \\
= & \int d \vec{z}_{1} d \vec{z}_{2} d \vec{z}_{3} F_{1}\left(p_{q}, p_{\bar{q}}, p_{g}, z_{0}, \vec{z}_{1}, \vec{z}_{2}, \vec{z}_{3}\right)^{\alpha} \frac{1}{2}\left(\operatorname{tr}\left(U_{1} U_{3}^{\dagger}\right) \operatorname{tr}\left(U_{3} U_{2}^{\dagger}\right)-N_{c} \operatorname{tr}\left(U_{1} U_{2}^{\dagger}\right)\right) \\
& +\int d \vec{z}_{1} d \vec{z}_{2} F_{2}\left(p_{q}, p_{\bar{q}}, p_{g}, z_{0}, \vec{z}_{1}, \vec{z}_{2}\right)^{\alpha} \frac{N_{c}^{2}-1}{2 N_{c}}\left(\operatorname{tr}\left(U_{1} U_{2}^{\dagger}\right)-N_{c}\right) .
\end{aligned}
$$

Here

$$
F_{2}\left(p_{q}, p_{\bar{q}}, p_{g}, z_{0}, \vec{z}_{1}, \vec{z}_{2}\right)^{\alpha}=\tilde{F}_{2}\left(p_{q}, p_{\bar{q}}, p_{g}, z_{0}, \vec{z}_{1}, \vec{z}_{2}\right)^{\alpha}+\int d \vec{z}_{3} F_{1}\left(p_{q}, p_{\bar{q}}, p_{g}, z_{0}, \vec{z}_{1}, \vec{z}_{2}, \vec{z}_{3}\right)^{\alpha},
$$

and

$$
\operatorname{tr}\left(U_{1} t^{a} U_{2}^{\dagger} t^{b}\right) U_{3}^{b a}=\frac{1}{2}\left(\operatorname{tr}\left(U_{1} U_{3}^{\dagger}\right) \operatorname{tr}\left(U_{3} U_{2}^{\dagger}\right)-N_{c} \operatorname{tr}\left(U_{1} U_{2}^{\dagger}\right)\right)+\frac{N_{c}^{2}-1}{2 N_{c}} \operatorname{tr}\left(U_{1} U_{2}^{\dagger}\right) .
$$

The functions $F_{1}$ and $\tilde{F}_{2}$ are the two above mentioned components of the impact factor, which we will calculate in the next two sections.

\section{$5 \quad$ Diagrams with the gluon crossing the shockwave}

Using eqs. (2.23)-(2.25), the sum of the first two diagrams in figure 2 can be represented in the following way

$F_{1}\left(z_{q}, z_{\bar{q}}, p_{g}, z_{0}, \vec{z}_{1}, \vec{z}_{2}, \vec{z}_{3}\right)^{\alpha}=\frac{i g}{(2 \pi)^{2}} \int d z_{1}^{+} d z_{1}^{-} d z_{2}^{+} d z_{2}^{-} d z_{3}^{+} d z_{3}^{-} \delta\left(z_{1}^{+}\right) \delta\left(z_{2}^{+}\right) \delta\left(z_{3}^{+}\right) L_{i \beta}^{j \alpha} R_{j}^{i \beta}$, 

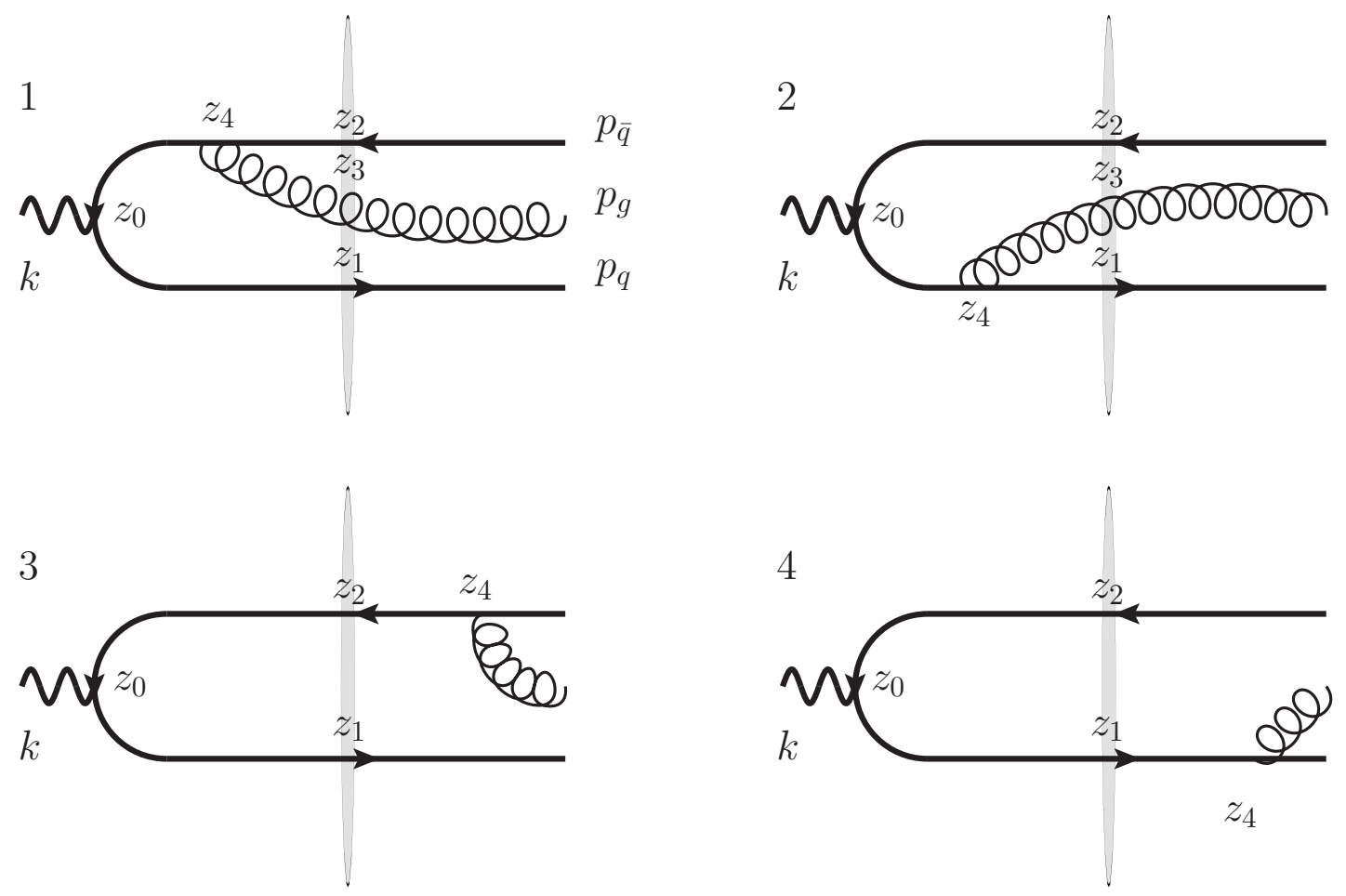

Figure 2. Impact factor for 3 jet production. The grey ellipse stands for the shockwave at $z^{+}=0$. The lines crossing the ellipse are calculated in the shock wave background (2.17), (2.23)-(2.25).

where

$$
\begin{aligned}
L_{i \beta}^{j \alpha}= & \int d z_{4} \frac{1}{\frac{\partial}{\partial z_{3}^{-}}} \frac{g_{\perp \beta \nu} z_{34}^{+}-z_{34 \perp \beta} n_{2 \nu}}{\left(z_{34}^{2}-i 0\right)^{2}} \\
& \times\left[\gamma^{+}\left(G_{0}\left(z_{10}\right) \gamma^{\alpha} G_{0}\left(z_{04}\right) \gamma^{\nu} G_{0}\left(z_{42}\right)+G_{0}\left(z_{14}\right) \gamma^{\nu} G_{0}\left(z_{40}\right) \gamma^{\alpha} G_{0}\left(z_{02}\right)\right) \gamma^{+}\right]_{i}^{j}
\end{aligned}
$$

and

$$
R_{j}^{i \beta}=p_{g}^{+} \theta\left(p_{g}^{+}\right) \frac{\varepsilon_{g}^{* \beta} e^{i p_{g} \cdot z_{3}+i p_{q} \cdot z_{1}+i p_{\bar{q}} \cdot z_{2}}}{\sqrt{2 p_{g}^{+} 2 p_{q}^{+} 2 p_{\bar{q}}^{+}}}\left[\gamma^{-} \gamma^{+} v_{p_{\bar{q}}} \otimes \bar{u}_{p_{q}} \gamma^{+} \gamma^{-}\right]_{j}^{i}
$$

Using the integral (2.29) and the fact that $z_{1,2,3}^{+}=0$ one can write

$$
\begin{aligned}
L_{i \beta}^{j \alpha}=\frac{2 z_{30}^{+}}{(2 \pi)^{4}} \frac{1}{\frac{\partial}{\partial z_{3}^{-}}}\left[\gamma^{+}\right. & \left(\frac{\hat{z}_{10} \gamma^{\alpha} \hat{z}_{30} \gamma_{\perp \beta} \hat{z}_{32}}{z_{30}^{4} z_{32}^{2} z_{20} z_{10}^{4}}+\frac{\hat{z}_{31} \gamma_{\perp \beta} \hat{z}_{30} \gamma^{\alpha} \hat{z}_{02}}{z_{31}^{2} z_{30}^{4} z_{01}{ }^{2} z_{02}^{4}}\right. \\
& \left.\left.+\frac{2}{z_{30}^{2}} \frac{\hat{z}_{10} \gamma^{\alpha} \hat{z}_{02}}{z_{20}{ }^{4} z_{10}^{4}}\left(\frac{z_{31 \perp \beta}}{z_{31}^{2}}-\frac{z_{32 \perp \beta}}{z_{32}^{2}}\right)\right) \gamma^{+}\right]_{i}^{j}
\end{aligned}
$$


Integrating w.r.t. $z_{3}^{-}$with the help of eq. (2.26) we have

$$
\begin{aligned}
L_{i \beta}^{j \alpha}= & \frac{-1}{(2 \pi)^{4}} \int_{\sigma}^{+\infty} \frac{d p^{+}}{p^{+}} e^{-i p^{+}\left(z_{30}^{-}-\frac{z_{30}^{2}+i 0}{2 z_{30}^{+}}\right)}\left[\gamma ^ { + } \left(\frac{i p^{+} \hat{z}_{10} \gamma^{\alpha}\left(\gamma^{-} z_{30}^{+}+\hat{z}_{30 \perp}\right) \gamma_{\perp \beta} \hat{z}_{32}}{\left(2 z_{30}^{+}\right) z_{32}^{2} z_{20}^{2} z_{10}^{4}}\right.\right. \\
& \left.\left.+\frac{i p^{+} \hat{z}_{31} \gamma_{\perp \beta}\left(\gamma^{-} z_{30}^{+}+\hat{z}_{30 \perp}\right) \gamma^{\alpha} \hat{z}_{02}}{z_{31}^{2}\left(2 z_{30}^{+}\right) z_{01}^{2} z_{02}^{4}}+2 \frac{\hat{z}_{10} \gamma^{\alpha} \hat{z}_{02}}{z_{20} z_{10}^{4}}\left(\frac{z_{31 \perp \beta}}{z_{31}^{2}}-\frac{z_{32 \perp \beta}}{z_{32}^{2}}\right)\right) \gamma^{+}\right]_{i}^{j},
\end{aligned}
$$

where $\sigma=e^{\eta}$ is the longitudinal cutoff (2.20). As a result,

$$
\begin{aligned}
F_{1}\left(p_{q}, p_{\bar{q}}, p_{g}, z_{0}, \vec{z}_{1}, \vec{z}_{2}, \vec{z}_{3}\right)^{\alpha}= & \theta\left(p_{g}^{+}-\sigma\right) \frac{i g}{(2 \pi)^{3}} \varepsilon_{g}^{* \beta} \frac{e^{i z_{0}^{-}\left(p_{q}^{+}+p_{\bar{q}}^{+}+p_{g}^{+}\right)-i \vec{p}_{q} \cdot \vec{z}_{1}-i \vec{p}_{\bar{q}} \cdot \vec{z}_{2}-i \vec{p}_{g} \cdot \vec{z}_{3}}}{\left(2 z_{0}^{+}\right)^{2} \sqrt{2 p_{q}^{+} 2 p_{\bar{q}}^{+} 2 p_{g}^{+}}} \\
& \times \bar{u}_{p_{q}} \gamma^{+}\left[\frac{z_{32 \perp}^{\omega}}{-\vec{z}_{32}^{2}}\left(i p_{q}^{+} \gamma^{-}-\gamma_{\perp}^{\sigma} \frac{\partial}{\partial z_{1 \perp}^{\sigma}}\right) \gamma^{\alpha}\left(i p_{g}^{+} \gamma^{-}-\gamma_{\perp}^{\rho} \frac{\partial}{\partial z_{3 \perp}^{\rho}}\right) \gamma_{\perp \beta} \gamma_{\perp \omega}\right. \\
& +\frac{\hat{z}_{31 \perp}}{\vec{z}_{31}^{2}} \gamma_{\perp \beta}\left(i p_{g}^{+} \gamma^{-}-\gamma_{\perp}^{\rho} \frac{\partial}{\partial z_{3 \perp}^{\rho}}\right) \gamma^{\alpha}\left(i p_{\bar{q}}^{+} \gamma^{-}-\gamma_{\perp}^{\sigma} \frac{\partial}{\partial z_{2 \perp}^{\sigma}}\right) \\
& \left.+2\left(\frac{z_{31 \perp \beta}}{\vec{z}_{31}^{2}}-\frac{z_{32 \perp \beta}}{\vec{z}_{32}^{2}}\right)\left(i p_{q}^{+} \gamma^{-}-\gamma_{\perp}^{\rho} \frac{\partial}{\partial z_{1 \perp}^{\rho}}\right) \gamma^{\alpha}\left(i p_{\bar{q}}^{+} \gamma^{-}-\gamma_{\perp}^{\sigma} \frac{\partial}{\partial z_{2 \perp}^{\sigma}}\right)\right] \gamma^{+} v_{p_{\bar{q}}} \\
& \times e^{-i \frac{p_{g}^{+} z_{30}^{2}+p_{q}^{+} \vec{z}_{10}^{2}+p_{\bar{q}}^{+} \vec{z}_{20}^{2}+i 0}{2 z_{0}^{+}}}
\end{aligned}
$$

Via eq. (2.30) we will calculate the Fourier transform of $F_{1}$

$$
F_{1}\left(p_{q}, p_{\bar{q}}, p_{g}, k, \vec{z}_{1}, \vec{z}_{2}, \vec{z}_{3}\right)^{\alpha}=\int d^{4} z_{0} e^{-i k \cdot z_{0}} F_{1}\left(p_{q}, p_{\bar{q}}, p_{g}, z_{0}, \vec{z}_{1}, \vec{z}_{2}, \vec{z}_{3}\right)^{\alpha}
$$

for the photon in our kinematics (2.9). Using our notation (2.8) and denoting

$$
Z_{123}=\sqrt{x_{q} x_{\bar{q}} \vec{z}_{12}^{2}+x_{q} x_{g} \vec{z}_{13}^{2}+x_{\bar{q}} x_{g} \vec{z}_{23}^{2}}
$$

we get

$$
\begin{aligned}
F_{1}\left(p_{q}, p_{\bar{q}}, p_{g}, k, \vec{z}_{1}, \vec{z}_{2}, \vec{z}_{3}\right)^{\alpha}= & -\frac{g \theta\left(p_{g}^{+}-\sigma\right)}{4 \pi k^{+}} \delta\left(k^{+}-p_{g}^{+}-p_{q}^{+}-p_{\bar{q}}^{+}\right) \varepsilon_{g \beta}^{*} \frac{e^{-i \vec{p}_{q} \cdot \vec{z}_{1}-i \vec{p}_{\bar{q}} \cdot \vec{z}_{2}-i \vec{p}_{g} \cdot \vec{z}_{3}}}{\sqrt{2 p_{q}^{+} 2 p_{\bar{q}}^{+} 2 p_{g}^{+}}} \\
& \times \bar{u}_{p_{q}} \gamma^{+}\left[\frac{z_{32 \perp}^{\omega}}{-\vec{z}_{32}^{2}}\left(i p_{q}^{+} \gamma^{-}-\gamma_{\perp}^{\sigma} \frac{\partial}{\partial z_{1 \perp}^{\sigma}}\right) \gamma^{\alpha}\left(i p_{g}^{+} \gamma^{-}-\gamma_{\perp}^{\rho} \frac{\partial}{\partial z_{3 \perp}^{\rho}}\right) \gamma_{\perp}^{\beta} \gamma_{\perp \omega}\right. \\
& +\frac{\hat{z}_{31 \perp}}{\vec{z}_{31}^{2}} \gamma_{\perp}^{\beta}\left(i p_{g}^{+} \gamma^{-}-\gamma_{\perp}^{\rho} \frac{\partial}{\partial z_{3 \perp}^{\rho}}\right) \gamma^{\alpha}\left(i p_{\bar{q}}^{+} \gamma^{-}-\gamma_{\perp}^{\sigma} \frac{\partial}{\partial z_{2 \perp}^{\sigma}}\right) \\
& \left.+2\left(\frac{z_{31 \perp}^{\beta}}{\vec{z}_{31}^{2}}-\frac{z_{32 \perp}^{\beta}}{\vec{z}_{32}^{2}}\right)\left(i p_{q}^{+} \gamma^{-}-\gamma_{\perp}^{\rho} \frac{\partial}{\partial z_{1 \perp}^{\rho}}\right) \gamma^{\alpha}\left(i p_{\bar{q}}^{+} \gamma^{-}-\gamma_{\perp}^{\sigma} \frac{\partial}{\partial z_{2 \perp}^{\sigma}}\right)\right] \\
& \times \gamma^{+} v_{p_{\bar{q}}} K_{0}\left(Q Z_{123}\right) .
\end{aligned}
$$

As before for the $\gamma \rightarrow q \bar{q}$ impact factor, we encounter contributions involving $\delta$ distributions for $\alpha=-$. Indeed, e.g.

$$
\frac{\partial^{2} K_{0}\left(Q Z_{123}\right)}{\partial z_{1 \perp}^{\sigma} \partial z_{3 \perp}^{\rho}}=Q^{2} \frac{\left(Q Z_{123} K_{0}^{\prime}\left(Q Z_{123}\right)\right)^{\prime}}{Q Z_{123}} \frac{\partial Z_{123}}{\partial z_{1 \perp}^{\sigma}} \frac{\partial Z_{123}}{\partial z_{3 \perp}^{\rho}}+Q Z_{123} K_{0}^{\prime}\left(Q Z_{123}\right) \frac{\partial^{2} \ln Z_{123}}{\partial z_{1 \perp}^{\sigma} \partial z_{3 \perp}^{\rho}}
$$


and from eq. (2.31) we obtain

$$
\frac{\left(Q Z_{123} K_{0}^{\prime}\left(Q Z_{123}\right)\right)^{\prime}}{Q Z_{123}}=K_{0}\left(Q Z_{123}\right)+4 \pi \delta\left(Q^{2} Z_{123}^{2}\right) \delta(\phi) .
$$

Again the term with the delta function gives a vanishing contribution to $M$ introduced in eq. (4.2) and we drop it. Then, using eq. (2.31) and the matrix elements (2.32), we get

$$
F_{1}^{+}=F_{1}^{-} \frac{2\left(k^{+}\right)^{2}}{Q^{2}}
$$

On one hand this implies the electromagnetic gauge invariance

$$
F_{1}\left(p_{q}, p_{\bar{q}}, p_{g}, k, \vec{z}_{1}, \vec{z}_{2}, \vec{z}_{3}\right)^{\alpha} k_{\alpha}=0,
$$

and on the second hand it gives the contribution to the impact factor for longitudinal photon (2.10)

$$
\begin{aligned}
& F_{1}\left(p_{q}, p_{\bar{q}}, p_{g}, k, \vec{z}_{1}, \vec{z}_{2}, \vec{z}_{3}\right)^{\alpha} \varepsilon_{L \alpha} \\
& =-\delta\left(k^{+}-p_{g}^{+}-p_{q}^{+}-p_{\bar{q}}^{+}\right) \theta\left(p_{g}^{+}-\sigma\right) Q g \frac{e^{-i \vec{p}_{q} \cdot \vec{z}_{1}-i \vec{p}_{\bar{q}} \cdot \vec{z}_{2}-i \vec{p}_{g} \cdot \vec{z}_{3}}}{\pi \sqrt{2 p_{g}^{+}}} K_{0}\left(Q Z_{123}\right) \\
& \quad \times \delta_{\lambda_{q},-\lambda_{\bar{q}}} \varepsilon_{g}^{* \beta}\left(i \lambda_{q} \epsilon^{\gamma \beta 3} x_{g}\left\{x_{\bar{q}} \frac{z_{31}^{\gamma}}{\vec{z}_{31}^{2}}+x_{q} \frac{z_{32}^{\gamma}}{\vec{z}_{32}^{2}}\right\}+\left\{\left(2 x_{q}+x_{g}\right) x_{\bar{q}} \frac{z_{31}^{\beta}}{\vec{z}_{31}^{2}}-\left(2 x_{\bar{q}}+x_{g}\right) x_{q} \frac{z_{32}^{\beta}}{\vec{z}_{32}^{2}}\right\}\right) .
\end{aligned}
$$

Expressing the vector products $e^{\gamma \beta 3} a^{\gamma} b^{\beta}=[\vec{a} \times \vec{b}]$ through the scalar products using eq. (2.16) we have

$$
\begin{aligned}
& F_{1}\left(p_{q}, p_{\bar{q}}, p_{g}, k, \vec{z}_{1}, \vec{z}_{2}, \vec{z}_{3}\right)^{\alpha} \varepsilon_{L \alpha} \\
& =\delta\left(k^{+}-p_{g}^{+}-p_{q}^{+}-p_{\bar{q}}^{+}\right) \theta\left(p_{g}^{+}-\sigma\right) 2 Q g \frac{e^{-i \vec{p}_{q} \cdot \vec{z}_{1}-i \vec{p}_{\bar{q}} \cdot \vec{z}_{2}-i \vec{p}_{g} \cdot \vec{z}_{3}}}{\pi \sqrt{2 p_{g}^{+}}} K_{0}\left(Q Z_{123}\right) \\
& \quad \times \delta_{\lambda_{q},-\lambda_{\bar{q}}}\left\{\left(x_{\bar{q}}+x_{g} \delta_{-s_{g} \lambda_{q}}\right) x_{q} \frac{\vec{z}_{32} \cdot \vec{\varepsilon}_{g}^{*}}{\vec{z}_{32}^{2}}-\left(x_{q}+x_{g} \delta_{-s_{g} \lambda_{\bar{q}}}\right) x_{\bar{q}} \frac{\vec{z}_{31} \cdot \vec{\varepsilon}_{g}^{*}}{\vec{z}_{31}^{2}}\right\} .
\end{aligned}
$$

For transverse photon (2.11) we get the following contribution to the impact factor

$$
\begin{aligned}
& F_{1}\left(p_{q}, p_{\bar{q}}, p_{g}, k, \vec{z}_{1}, \vec{z}_{2}, \vec{z}_{3}\right)^{\alpha} \varepsilon_{T \alpha} \\
& =2 i g Q \delta\left(k^{+}-p_{g}^{+}-p_{q}^{+}-p_{\bar{q}}^{+}\right) \theta\left(p_{g}^{+}-\sigma\right) \frac{e^{-i \vec{p}_{q} \cdot \vec{z}_{1}-i \vec{p}_{\bar{q}} \cdot \vec{z}_{2}-i \vec{p}_{g} \cdot \vec{z}_{3}}}{\pi Z_{123} \sqrt{2 p_{g}^{+}}} \\
& \quad \times \delta_{\lambda_{q},-\lambda_{\bar{q}}} K_{1}\left(Q Z_{123}\right)\left\{-\frac{\left(\vec{z}_{23} \cdot \vec{\varepsilon}_{g}^{*}\right)\left(\vec{z}_{13} \cdot \vec{\varepsilon}_{T}\right)}{\vec{z}_{23}{ }^{2}} x_{q}\left(x_{q}-\delta_{s \lambda_{\bar{q}}}\right)\left(x_{\bar{q}}+x_{g} \delta_{-s_{g} \lambda_{q}}\right)\right. \\
& \left.\quad-\frac{\left(\vec{z}_{23} \cdot \vec{\varepsilon}_{g}^{*}\right)\left(\vec{z}_{23} \cdot \vec{\varepsilon}_{T}\right)}{\vec{z}_{23}{ }^{2}} x_{q} x_{\bar{q}}\left(x_{\bar{q}}+x_{g} \delta_{-s_{g} \lambda_{q}}-\delta_{s \lambda_{q}}\right)\right\}-(q \leftrightarrow \bar{q}),
\end{aligned}
$$

where

$$
(q \leftrightarrow \bar{q}) \equiv\left(\lambda_{q}, x_{q}, \vec{z}_{1}, \vec{p}_{q} \leftrightarrow \lambda_{\bar{q}}, x_{\bar{q}}, \vec{z}_{2}, \vec{p}_{\bar{q}}\right)
$$

One can check that the results (5.15) and (5.16) are compatible with the wave function derived in ref. [49]. 


\section{Diagrams without the gluon crossing the shockwave}

Here we will calculate $\tilde{F}_{2}$ (4.3), which gives the contribution from diagrams 3 and 4 in figure 2. It reads

$$
\tilde{F}_{2}\left(z_{q}, z_{\bar{q}}, p_{g}, z_{0}, \vec{z}_{1}, \vec{z}_{2}\right)^{\alpha}=\frac{i g}{4} \int d z_{1}^{+} d z_{1}^{-} d z_{2}^{+} d z_{2}^{-} \delta\left(z_{1}^{+}\right) \delta\left(z_{2}^{+}\right) L_{i}^{\prime j \alpha} R_{j}^{\prime i}
$$

where

$$
L_{i}^{\prime j \alpha}=\left[\gamma^{+} G_{0}\left(z_{10}\right) \gamma^{\alpha} G_{0}\left(z_{02}\right) \gamma^{+}\right]_{i}^{j}
$$

and

$$
\begin{aligned}
R_{j}^{i \beta}= & \int \theta\left(z_{4}^{+}\right) d^{4} z_{4} \frac{\varepsilon_{g}^{* \beta} e^{i p_{g} \cdot z_{4}}}{\sqrt{2 p_{g}^{+} 2 p_{q}^{+} 2 p_{\bar{q}}^{+}}} \\
& \times\left[\gamma^{-} \gamma^{+}\left(e^{i p_{q} \cdot z_{1}+i p_{\bar{q}} \cdot z_{4}} G_{0}\left(z_{24}\right) \gamma_{\beta} v_{p_{\bar{q}}} \otimes \bar{u}_{p_{q}}+e^{i p_{q} \cdot z_{4}+i p_{\bar{q}} \cdot z_{2}} v_{p_{\bar{q}}} \otimes \bar{u}_{p_{q}} \gamma_{\beta} G_{0}\left(z_{41}\right)\right) \gamma^{+} \gamma^{-}\right]_{j}^{i}
\end{aligned}
$$

Integrating eq. (6.3) w.r.t. $z_{4}$, we get

$$
\begin{aligned}
R_{j}^{\prime i}= & -i \frac{\varepsilon_{g}^{* \beta}}{\sqrt{2 p_{g}^{+} 2 p_{q}^{+} 2 p_{\bar{q}}^{+}}} e^{i p_{q}^{+} z_{1}^{-}-i \vec{p}_{q} \cdot \vec{z}_{1}+i p_{\bar{q}}^{+} z_{2}^{-}-i \vec{p}_{\vec{q}} \cdot \vec{z}_{2}}\left[\gamma ^ { - } \gamma ^ { + } \left(e^{i p_{g}^{+} z_{2}^{-}-i \vec{p}_{g} \cdot \vec{z}_{2}} \frac{\left(\hat{p}_{\bar{q}}+\hat{p}_{g}\right)}{\left(p_{\bar{q}}+p_{g}\right)^{2}} \gamma_{\beta} v_{p_{\bar{q}}} \otimes \bar{u}_{p_{q}}\right.\right. \\
& \left.\left.-v_{p_{\bar{q}}} \otimes \bar{u}_{p_{q}} \gamma_{\beta} \frac{\left(\hat{p}_{q}+\hat{p}_{g}\right)}{\left(p_{q}+p_{g}\right)^{2}} e^{i p_{g}^{+} z_{1}^{-}-i \vec{p}_{g} \cdot \vec{z}_{1}}\right) \gamma^{+} \gamma^{-}\right]_{j}^{i} .
\end{aligned}
$$

As a result,

$$
\begin{aligned}
\tilde{F}_{2}\left(p_{q}, p_{\bar{q}}, p_{g}, z_{0}, \vec{z}_{1}, \vec{z}_{2}\right)^{\alpha} & \\
= & \theta\left(p_{g}^{+}-\sigma\right) \frac{g}{16 \pi^{2}} \frac{\varepsilon_{g}^{* \beta} \theta\left(p_{g}^{+}\right)}{\sqrt{2 p_{g}^{+} 2 p_{q}^{+} 2 p_{\bar{q}}^{+}}} \frac{1}{\left(z_{0}^{+}\right)^{2}} e^{i\left(p_{\bar{q}}^{+}+p_{q}^{+}+p_{g}^{+}\right) z_{0}^{-}-i \vec{p}_{q} \cdot \vec{z}_{1}-i \vec{p}_{\bar{q}} \cdot \vec{z}_{2}} \\
& \times\left\{-e^{-i \vec{p}_{g} \cdot \vec{z}_{2}} \bar{u}_{p_{q}} \gamma^{+}\left(i p_{q}^{+} \gamma^{-}-\gamma_{\perp}^{\sigma} \frac{\partial}{\partial z_{1 \perp}^{\sigma}}\right) \gamma^{\alpha}\left(i\left(p_{g}^{+}+p_{\bar{q}}^{+}\right) \gamma^{-}-\gamma_{\perp}^{\sigma} \frac{\partial}{\partial z_{2 \perp}^{\sigma}}\right) \gamma^{+} \frac{\left(\hat{p}_{\bar{q}}+\hat{p}_{g}\right)}{\left(p_{\bar{q}}+p_{g}\right)^{2}} \gamma_{\beta} v_{p_{\bar{q}}}\right. \\
& \times e^{-i k^{+} \frac{\left(x_{g}+x_{\bar{q}}\right) \vec{z}_{20}^{2}+x_{q} \vec{z}_{10}^{2}+i 0}{2 z_{0}^{+}}}+e^{-i \vec{p}_{g} \vec{z}_{1}} \bar{u}_{p_{q}} \gamma_{\beta} \frac{\left(\hat{p}_{q}+\hat{p}_{g}\right)}{\left(p_{q}+p_{g}\right)^{2}} \gamma^{+}\left(i\left(p_{g}^{+}+p_{q}^{+}\right) \gamma^{-}-\gamma_{\perp}^{\sigma} \frac{\partial}{\partial z_{1 \perp}^{\sigma}}\right) \\
& \left.\times \gamma^{\alpha}\left(i p_{\bar{q}}^{+} \gamma^{-}-\gamma_{\perp}^{\sigma} \frac{\partial}{\partial z_{2 \perp}^{\sigma}}\right) \gamma^{+} v_{p_{\bar{q}}} e^{-i k^{+} \frac{x_{\bar{q}} \vec{z}_{20}^{2}+\left(x_{g}+x_{q}\right) \vec{z}_{10}^{2}+i 0}{2 z_{0}^{+}}}\right\} .
\end{aligned}
$$

Next we calculate the Fourier transform of $\tilde{F}_{2}$

$$
\tilde{F}_{2}\left(p_{q}, p_{\bar{q}}, p_{g}, k, \vec{z}_{1}, \vec{z}_{2}\right)^{\alpha}=\int d^{4} z_{0} e^{-i k \cdot z_{0}} \tilde{F}_{2}\left(p_{q}, p_{\bar{q}}, p_{g}, z_{0}, \vec{z}_{1}, \vec{z}_{2}\right)^{\alpha}
$$


for the photon in our kinematics (2.9)

$$
\begin{aligned}
\tilde{F}_{2}\left(p_{q}, p_{\bar{q}}, p_{g}, k, \vec{z}_{1}, \vec{z}_{2}\right)^{\alpha} & \\
= & \frac{i g}{2 k^{+}} \theta\left(p_{g}^{+}-\sigma\right) \delta\left(k^{+}-p_{g}^{+}-p_{q}^{+}-p_{\bar{q}}^{+}\right) \varepsilon_{g}^{* \beta} \frac{e^{-i \vec{p}_{q} \cdot \vec{z}_{1}-i \vec{p}_{\bar{q}} \cdot \vec{z}_{2}}}{\sqrt{2 p_{g}^{+} 2 p_{q}^{+} 2 p_{\bar{q}}^{+}}} \\
& \times\left[-e^{-i \vec{p}_{g} \cdot \vec{z}_{2}} \bar{u}_{p_{q}} \gamma^{+}\left(i p_{q}^{+} \gamma^{-}-\gamma_{\perp}^{\sigma} \frac{\partial}{\partial z_{1 \perp}^{\sigma}}\right) \gamma^{\alpha}\left(i\left(p_{g}^{+}+p_{\bar{q}}^{+}\right) \gamma^{-}-\gamma_{\perp}^{\sigma} \frac{\partial}{\partial z_{2 \perp}^{\sigma}}\right) \gamma^{+}\right. \\
& \times \frac{\left(\hat{p}_{\bar{q}}+\hat{p}_{g}\right)}{\left(p_{\bar{q}}+p_{g}\right)^{2}} \gamma_{\beta} v_{p_{\bar{q}}} K_{0}\left(Q Z_{122}\right)+e^{-i \vec{p}_{g} \cdot \vec{z}_{1}} \bar{u}_{p_{q}} \gamma_{\beta} \frac{\left(\hat{p}_{q}+\hat{p}_{g}\right)}{\left(p_{q}+p_{g}\right)^{2}} \\
& \left.\times \gamma^{+}\left(i\left(p_{g}^{+}+p_{q}^{+}\right) \gamma^{-}-\gamma_{\perp}^{\sigma} \frac{\partial}{\partial z_{1 \perp}^{\sigma}}\right) \gamma^{\alpha}\left(i p_{\bar{q}}^{+} \gamma^{-}-\gamma_{\perp}^{\sigma} \frac{\partial}{\partial z_{2 \perp}^{\sigma}}\right) \gamma^{+} v_{p_{\bar{q}}} K_{0}\left(Q Z_{121}\right)\right] .
\end{aligned}
$$

In the arguments of the McDonald functions we encounter the following structures

$$
\begin{aligned}
& Z_{122}=\left.Z_{123}\right|_{z_{3} \rightarrow z_{2}}=\sqrt{x_{q}\left(x_{\bar{q}}+x_{g}\right) \vec{z}_{12}^{2}}=\sqrt{x_{q}\left(1-x_{q}\right) \vec{z}_{12}^{2}}, \\
& Z_{121}=\left.Z_{123}\right|_{z_{3} \rightarrow z_{1}}=\sqrt{\left(x_{q}+x_{g}\right) x_{\bar{q}} \vec{z}_{21}^{2}}=\sqrt{\left(1-x_{\bar{q}}\right) x_{\bar{q}} \vec{z}_{21}^{2}} .
\end{aligned}
$$

Again using the Bessel equation (2.31) as well as the matrix elements (2.32) and (2.33), then dropping the corresponding $\delta$ distributions we can check the electromagnetic gauge invariance

$$
\tilde{F}_{2}\left(p_{q}, p_{\bar{q}}, p_{g}, k, \vec{z}_{1}, \vec{z}_{2}\right)^{\alpha} k_{\alpha}=0 .
$$

Then taking into account Dirac equation and the gauge condition (2.12), we get the contribution to the impact factor for longitudinal photon (2.10)

$$
\begin{aligned}
& \tilde{F}_{2}\left(p_{q}, p_{\bar{q}}, p_{g}, k, \vec{z}_{1}, \vec{z}_{2}\right)^{\alpha} \varepsilon_{L \alpha} \\
& =4 i g Q \theta\left(p_{g}^{+}-\sigma\right) \delta\left(k^{+}-p_{g}^{+}-p_{q}^{+}-p_{\bar{q}}^{+}\right) \frac{e^{-i \vec{p}_{q} \cdot \vec{z}_{1}-i \vec{p}_{\bar{q}} \cdot \vec{z}_{2}}}{\sqrt{2 p_{g}^{+}}} \\
& \quad \times \delta_{\lambda_{q},-\lambda_{\bar{q}}} \frac{x_{q}\left(x_{g}+x_{\bar{q}}\right)\left(\delta_{-s_{g} \lambda_{q}} x_{g}+x_{\bar{q}}\right)}{x_{\bar{q}} x_{g}} \frac{\vec{P}_{\bar{q}} \cdot \vec{\varepsilon}_{g}^{*}}{\vec{P}_{\bar{q}}^{2}} e^{-i \vec{p}_{g} \cdot \vec{z}_{2}} K_{0}\left(Q Z_{122}\right)-(q \leftrightarrow \bar{q}),
\end{aligned}
$$

where $P_{q, \bar{q}}$ are defined in eq. (2.14). For transverse photons (2.11) we have

$$
\begin{aligned}
& \tilde{F}_{2}\left(p_{q}, p_{\bar{q}}, p_{g}, k, \vec{z}_{1}, \vec{z}_{2}\right)^{\alpha} \varepsilon_{T \alpha} \\
& =-4 g \theta\left(p_{g}^{+}-\sigma\right) \delta\left(k^{+}-p_{g}^{+}-p_{q}^{+}-p_{\bar{q}}^{+}\right) \frac{e^{-i \vec{p}_{q} \cdot \vec{z}_{1}-i \vec{p}_{\bar{q}} \cdot \vec{z}_{2}}}{\sqrt{2 p_{g}^{+}}} \delta_{\lambda_{q},-\lambda_{\bar{q}}} \\
& \quad \times \frac{\left(\delta_{\lambda_{\bar{q}} s}-x_{q}\right)\left(\delta_{-s_{g} \lambda_{q}} x_{g}+x_{\bar{q}}\right)}{x_{\bar{q}} x_{g}} \frac{\vec{P}_{\bar{q}} \cdot \vec{\varepsilon}_{g}^{*}}{\vec{P}_{\bar{q}}^{2}} \frac{\vec{z}_{12} \cdot \vec{\varepsilon}_{T}}{\vec{z}_{12}^{2}} Q Z_{122} K_{1}\left(Q Z_{122}\right) e^{-i \vec{p}_{g} \cdot \vec{z}_{2}}-(q \leftrightarrow \bar{q}) .
\end{aligned}
$$

\section{Impact factor for interaction with a color dipole}

In the 2- and 3-gluon approximations (BFKL and BKP) of exchanges in $t$-channel one needs the Green function obeying the linear equation. In the color singlet channel, the subtracted color dipole is the operator that plays this role

$$
\mathbf{U}_{12}=\frac{1}{N_{c}} \operatorname{tr}\left(U_{1} U_{2}^{\dagger}\right)-1
$$


The operator appearing in eq. (4.3) can be rewritten as

$$
\operatorname{tr}\left(U_{1} U_{3}^{\dagger}\right) \operatorname{tr}\left(U_{3} U_{2}^{\dagger}\right)-N_{c} \operatorname{tr}\left(U_{1} U_{2}^{\dagger}\right)=N_{c}^{2}\left(\mathbf{U}_{32}+\mathbf{U}_{13}-\mathbf{U}_{12}+\mathbf{U}_{32} \mathbf{U}_{13}\right) .
$$

Therefore in the 2- and 3-gluon approximations in which we neglect $\mathbf{U}_{32} \mathbf{U}_{13}$, eq. (4.2) reads

$$
\begin{aligned}
M^{\alpha} \stackrel{\mathrm{g}^{3}}{=} & \int d \vec{z}_{1} d \vec{z}_{2} \mathbf{U}_{12}\left\{\frac{N_{c}^{2}-1}{2} \tilde{F}_{2}\left(p_{q}, p_{\bar{q}}, p_{g}, z_{0}, \vec{z}_{1}, \vec{z}_{2}\right)^{\alpha}-\frac{1}{2} \int d \vec{z}_{3} F_{1}\left(p_{q}, p_{\bar{q}}, p_{g}, z_{0}, \vec{z}_{1}, \vec{z}_{2}, \vec{z}_{3}\right)^{\alpha}\right\} \\
& +\frac{1}{2} N_{c}^{2} \int d \vec{z}_{1} d \vec{z}_{3} \mathbf{U}_{13} \int d \vec{z}_{2} F_{1}\left(p_{q}, p_{\bar{q}}, p_{g}, z_{0}, \vec{z}_{1}, \vec{z}_{2}, \vec{z}_{3}\right)^{\alpha} \\
& +\frac{1}{2} N_{c}^{2} \int d \vec{z}_{2} d \vec{z}_{3} \mathbf{U}_{32} \int d \vec{z}_{1} F_{1}\left(p_{q}, p_{\bar{q}}, p_{g}, z_{0}, \vec{z}_{1}, \vec{z}_{2}, \vec{z}_{3}\right)^{\alpha},
\end{aligned}
$$

which we write as

$$
M^{\alpha} \stackrel{\mathrm{g}^{3}}{=} \frac{1}{2} \int d \vec{z}_{1} d \vec{z}_{2} \mathbf{U}_{12}\left\{\tilde{F}_{1}\left(p_{q}, p_{\bar{q}}, p_{g}, z_{0}, \vec{z}_{1}, \vec{z}_{2}\right)^{\alpha}+\left(N_{c}^{2}-1\right) \tilde{F}_{2}\left(p_{q}, p_{\bar{q}}, p_{g}, z_{0}, \vec{z}_{1}, \vec{z}_{2}\right)^{\alpha}\right\}
$$

where

$$
\begin{aligned}
\tilde{F}_{1}\left(p_{q}, p_{\bar{q}}, p_{g}, z_{0}, \vec{z}_{1}, \vec{z}_{2}\right)^{\alpha}= & \int d \overrightarrow{z_{3}}\left[N_{c}^{2} F_{1}\left(p_{q}, p_{\bar{q}}, p_{g}, z_{0}, \vec{z}_{1}, \vec{z}_{3}, \vec{z}_{2}\right)^{\alpha}\right. \\
& \left.+N_{c}^{2} F_{1}\left(p_{q}, p_{\bar{q}}, p_{g}, z_{0}, \vec{z}_{3}, \vec{z}_{2}, \vec{z}_{1}\right)^{\alpha}-F_{1}\left(p_{q}, p_{\bar{q}}, p_{g}, z_{0}, \vec{z}_{1}, \vec{z}_{2}, \vec{z}_{3}\right)^{\alpha}\right] .
\end{aligned}
$$

The integrals required in order to calculate expression (7.5) are discussed in appendix A. Using (A.1) and (A.3) for longitudinal photon (2.10) we have

$$
\begin{aligned}
& \int d \vec{z}_{3} F_{1}\left(p_{q}, p_{\bar{q}}, p_{g}, k, \vec{z}_{1}, \vec{z}_{2}, \vec{z}_{3}\right)^{\alpha} \varepsilon_{L \alpha} \\
& =-\delta_{\lambda_{q},-\lambda_{\bar{q}}} \delta\left(k^{+}-p_{g}^{+}-p_{q}^{+}-p_{\bar{q}}^{+}\right) \theta\left(p_{g}^{+}-\sigma\right) \\
& \quad \times \frac{2 Q g}{\sqrt{2 p_{g}^{+}}} e^{-i \vec{p}_{q} \cdot \vec{z}_{1}-i \vec{p}_{\vec{q}} \vec{z}_{2}}\left(x_{\bar{q}}+x_{g} \delta_{-s_{g} \lambda_{q}}\right) x_{q} e^{-i \vec{p}_{g} \cdot \vec{z}_{2}} \int_{0}^{1} d \alpha e^{\alpha \frac{i x_{q}\left(\vec{z}_{1} \cdot \vec{p}_{g}\right)}{x_{\bar{q}}+x_{q}}} \\
& \quad \times\left(i\left(\vec{p}_{g} \cdot \vec{\varepsilon}_{g}^{*}\right) \frac{Q_{g}(\alpha) Z_{q \bar{q} g} K_{1}\left(Q_{g}(\alpha) Z_{q \bar{q} g}\right)}{x_{g}\left(1-x_{g}\right) Q^{2}+\alpha \vec{p}_{g}^{2}}+\frac{x_{q}}{x_{q}+x_{\bar{q}}}\left(\vec{z}_{21} \cdot \vec{\varepsilon}_{g}^{*}\right) K_{0}\left(Q_{g}(\alpha) Z_{q \bar{q} g}\right)\right)-(q \leftrightarrow \bar{q}),
\end{aligned}
$$

and

$$
\begin{aligned}
& \int d \vec{z}_{3} F_{1}\left(p_{q}, p_{\bar{q}}, p_{g}, k, \vec{z}_{3}, \vec{z}_{2}, \vec{z}_{1}\right)^{\alpha} \varepsilon_{L \alpha} \\
& =\delta_{\lambda_{q},-\lambda_{\bar{q}}} \delta\left(k^{+}-p_{g}^{+}-p_{q}^{+}-p_{\bar{q}}^{+}\right) \theta\left(p_{g}^{+}-\sigma\right) \frac{2 Q g}{\sqrt{2 p_{g}^{+}}} \\
& \quad \times e^{-i \vec{p}_{\bar{q}} \cdot \vec{z}_{2}-i \vec{p}_{g} \cdot \vec{z}_{1}}\left[2 x_{q}\left(x_{\bar{q}}+x_{g} \delta_{-s_{g} \lambda_{q}}\right) \frac{\vec{z}_{12} \cdot \vec{\varepsilon}_{g}^{*}}{\vec{z}_{12}^{2}} e^{-i \frac{x_{\bar{q}}\left(\vec{p} q \cdot \vec{z}_{2}\right)+x_{g}\left(\vec{p}_{q} \cdot \vec{z}_{1}\right)}{x_{g}+x_{\bar{q}}}} \frac{Q_{q}(1) Z_{\bar{q} g} K_{1}\left(Z_{\bar{q} g} Q_{q}(1)\right)}{x_{q}\left(1-x_{q}\right) Q^{2}+\vec{p}_{q}^{2}}\right. \\
& -\left(x_{q}+x_{g} \delta_{-s_{g} \lambda_{\bar{q}}}\right) x_{\bar{q}} e^{-i \vec{p}_{q} \cdot \vec{z}_{1}} \int_{0}^{1} d \alpha e^{\frac{\alpha \frac{i \bar{x}_{\bar{q}}\left(\vec{z}_{12} \cdot \vec{p}_{q}\right)}{x_{g}+x_{\bar{q}}}}{x^{2}}} \\
& \left.\quad \times\left(i\left(\vec{p}_{q} \cdot \vec{\varepsilon}_{g}^{*}\right) \frac{Q_{q}(\alpha) Z_{\bar{q} g q}(\alpha)}{x_{q}\left(1-x_{q}\right) Q^{2}+\alpha \vec{p}_{q}^{2}} K_{1}\left(Q_{q}(\alpha) Z_{\bar{q} g q}\right)-\frac{x_{\bar{q}}}{x_{\bar{q}}+x_{g}}\left(\vec{z}_{21} \cdot \vec{\varepsilon}_{g}^{*}\right) K_{0}\left(Q_{q}(\alpha) Z_{\bar{q} g q}\right)\right)\right] .
\end{aligned}
$$


Note that

$$
\int d \vec{z}_{3} F_{1}\left(p_{q}, p_{\bar{q}}, p_{g}, k, \vec{z}_{1}, \vec{z}_{3}, \vec{z}_{2}\right)^{\alpha} \varepsilon_{L \alpha}=-\left.\int d \vec{z}_{3} F_{1}\left(p_{q}, p_{\bar{q}}, p_{g}, k, \vec{z}_{3}, \vec{z}_{2}, \vec{z}_{1}\right)^{\alpha} \varepsilon_{L \alpha}\right|_{q \leftrightarrow \bar{q}}
$$

which allows one to evaluate the third integral involved in eq. (7.5). In eqs. (7.6), (7.7) we use the notations

$$
Z_{i j}=\sqrt{\frac{x_{i} x_{j}}{x_{i}+x_{j}} \vec{z}_{12}^{2}}, \quad Z_{i j k}=\sqrt{x_{i} \frac{x_{j}+(1-\alpha) x_{i} x_{k}}{x_{j}+x_{i}} \vec{z}_{21}^{2}}, \quad Q_{i}(\alpha)=\sqrt{Q^{2}+\frac{\alpha \vec{p}_{i}^{2}}{\left(1-x_{i}\right) x_{i}}} .
$$

For forward production $\vec{p}_{q}=\vec{p}_{\bar{q}}=\vec{p}_{g}=0$ one can simplify these expressions with the help of eq. (A.6)

$$
\begin{aligned}
\left.\tilde{F}_{1}\left(p_{q}, p_{\bar{q}}, p_{g}, k, \vec{z}_{1}, \vec{z}_{2}\right)^{\alpha} \varepsilon_{L \alpha}\right|_{\vec{p}_{q}=\vec{p}_{\bar{q}}=\vec{p}_{g}=0} \\
=-\delta\left(k^{+}-p_{g}^{+}-p_{q}^{+}-p_{\bar{q}}^{+}\right) \theta\left(p_{g}^{+}-\sigma\right) \\
\quad \times \delta_{\lambda_{q},-\lambda_{\bar{q}}} \frac{4 g N_{c}^{2}}{\sqrt{2 p_{g}^{+}}} \frac{\left(\vec{z}_{21} \cdot \vec{\varepsilon}_{g}^{*}\right)}{\vec{z}_{21}^{2}}\left\{-\frac{Z_{q \bar{q}} K_{1}\left(Q Z_{q \bar{q}}\right)}{2 N_{c}^{2} x_{g}}+\left(x_{\bar{q}}+x_{g} \delta_{-s_{g} \lambda_{q}}\right)\right. \\
\left.\quad \times\left(\frac{Z_{g \bar{q}} K_{1}\left(Z_{\bar{q} g} Q\right)}{\left(1-x_{q}\right)}-\frac{Z_{q g} K_{1}\left(Q Z_{q g}\right)}{x_{\bar{q}}}+Z_{122} K_{1}\left(Q Z_{122}\right)\left(\frac{1}{x_{\bar{q}}}+\frac{1}{N_{c}^{2} x_{g}}\right)\right)\right\}-(q \leftrightarrow \bar{q}) .
\end{aligned}
$$

For transverse photon (2.11) we can rewrite (5.16) as

$$
\begin{aligned}
& F_{1}\left(p_{q}, p_{\bar{q}}, p_{g}, k, \vec{z}_{1}, \vec{z}_{2}, \vec{z}_{3}\right)^{\alpha} \varepsilon_{T \alpha} \\
& =-2 i g \delta\left(k^{+}-p_{g}^{+}-p_{q}^{+}-p_{\bar{q}}^{+}\right) \theta\left(p_{g}^{+}-\sigma\right) \frac{e^{-i \vec{p}_{q} \cdot \vec{z}_{1}-i \vec{p}_{\bar{q}} \cdot \vec{z}_{2}-i \vec{p}_{g} \cdot \vec{z}_{3}}}{\pi \sqrt{2 p_{g}^{+}}} \varepsilon_{g}^{* j} \varepsilon_{T}^{u} \delta_{\lambda_{q},-\lambda_{\bar{q}}} \frac{z_{23}{ }_{23}{ }^{2}}{z^{2}} \\
& \quad \times\left(\nabla_{1}^{u}\left(\delta_{s \lambda_{\bar{q}}}\left(x_{\bar{q}}+x_{g} \delta_{-s_{g} \lambda_{q}}\right)-x_{q} \delta_{s \lambda_{q}}\right)-x_{q} \nabla_{3}^{u} \delta_{s \lambda_{q}} \delta_{-s_{g} \lambda_{\bar{q}}}\right) K_{0}\left(Q Z_{123}\right)-(q \leftrightarrow \bar{q}) .
\end{aligned}
$$

Then using (A.3) and (A.8) we have

$$
\begin{aligned}
\int & d \vec{z}_{3} F_{1}\left(p_{q}, p_{\bar{q}}, p_{g}, k, \vec{z}_{1}, \vec{z}_{2}, \vec{z}_{3}\right)^{\alpha} \varepsilon_{T \alpha} \\
= & 2 i g \delta\left(k^{+}-p_{g}^{+}-p_{q}^{+}-p_{\bar{q}}^{+}\right) \theta\left(p_{g}^{+}-\sigma\right) \varepsilon_{g}^{* j} \varepsilon_{T}^{u} \\
& \times \delta_{\lambda_{q},-\lambda_{\bar{q}}} \frac{e^{-i \vec{p}_{q} \cdot \vec{z}_{1}-i \vec{p}_{\bar{q}} \cdot \vec{z}_{2}}}{\sqrt{2 p_{g}^{+}}} \int_{0}^{1} d \alpha\left[e ^ { - \frac { i \alpha x _ { q } ( \vec { p } _ { g } \cdot \vec { z } _ { 1 2 } ) } { x _ { \overline { q } } x _ { q } } - i \vec { p } _ { g } \cdot \vec { z } _ { 2 } } \left\{\left(\delta_{s \lambda_{\bar{q}}}\left(x_{\bar{q}}+x_{g} \delta_{-s_{g} \lambda_{q}}\right)-x_{q} \delta_{s \lambda_{q}}\right)\right.\right. \\
& \times\left(K_{0}\left(Q_{g}(\alpha) Z_{q \bar{q} g}\right)\left(\frac{i p_{g}{ }^{j} z_{12}}{\vec{z}_{12}{ }^{2}} \frac{Z_{q \bar{q} g}{ }^{2}}{x_{g}\left(1-x_{g}\right)}-\alpha\left(\frac{x_{q}}{x_{q}+x_{\bar{q}}}\right)^{2} i p_{g}{ }^{u} z_{12}{ }^{j}+\frac{x_{q}}{x_{q}+x_{\bar{q}}} \delta^{j u}\right)\right. \\
& \left.-\frac{x_{q}}{x_{q}+x_{\bar{q}}} Q_{g}(\alpha) Z_{q \bar{q} g} K_{1}\left(Q_{g}(\alpha) Z_{q \bar{q} g}\right)\left(\frac{z_{12}{ }^{j} z_{12}{ }^{u}}{\vec{z}_{12}{ }^{2}}+\frac{\alpha p_{g}{ }^{j} p_{g}{ }^{u}}{x_{g}\left(1-x_{g}\right) Q^{2}+\alpha \vec{p}_{g}^{2}}\right)\right)-x_{q} \delta_{s \lambda_{q}} \delta_{-s_{g} \lambda_{\bar{q}}} \\
& \times\left(Z_{q \bar{q} g} Q_{g}(\alpha) K_{1}\left(Q_{g}(\alpha) Z_{q \bar{q} g}\right)\left(\frac{z_{12}{ }^{j} z_{12}}{\vec{z}_{12}{ }^{2}} \frac{(1-\alpha) x_{q} x_{g}}{x_{\bar{q}}+(1-\alpha) x_{q} x_{g}}+\frac{\alpha p_{g}{ }^{j} p_{g}{ }^{u}}{x_{g}\left(1-x_{g}\right) Q^{2}+\alpha \vec{p}_{g}^{2}}\right)\right. \\
& \left.\left.\left.-K_{0}\left(Q_{g}(\alpha) Z_{q \bar{q} g}\right)\left(\delta^{j u}-\alpha \frac{x_{q} z_{12}{ }^{j} i p_{g}{ }^{u}}{x_{q}+x_{\bar{q}}}+(1-\alpha) \frac{x_{q}}{x_{q}+x_{\bar{q}}} i p_{g}{ }^{j} z_{12}{ }^{u}\right)\right)\right\}-(q \leftrightarrow \bar{q})\right](7.12)
\end{aligned}
$$


as well as

$$
\begin{aligned}
\int & d \vec{z}_{3} F_{1}\left(p_{q}, p_{\bar{q}}, p_{g}, k, \vec{z}_{3}, \vec{z}_{2}, \vec{z}_{1}\right)^{\alpha} \varepsilon_{T \alpha} \\
= & 2 i g \delta\left(k^{+}-p_{g}^{+}-p_{q}^{+}-p_{\bar{q}}^{+}\right) \theta\left(p_{g}^{+}-\sigma\right) \varepsilon_{g}^{* j} \varepsilon_{T}^{u} \delta_{\lambda_{q},-\lambda_{\bar{q}}} \frac{e^{-i \vec{p}_{g} \cdot \vec{z}_{1}-i \vec{p}_{\bar{q}} \cdot \vec{z}_{2}}}{\sqrt{2 p_{g}^{+}}} \\
& \times\left[2 e ^ { - i \frac { x _ { \overline { q } } ( \vec { p } _ { q } \cdot \vec { z } _ { 2 } ) + x _ { g } ( \vec { p } _ { q } \cdot \vec { z } _ { 1 } ) } { x _ { \overline { q } + x _ { g } } } } \left\{\left(\delta_{s \lambda_{\bar{q}}}\left(x_{\bar{q}}+x_{g} \delta_{-s_{g} \lambda_{q}}\right)-x_{q} \delta_{s \lambda_{q}}\right) \frac{z_{12}{ }^{j} i p_{q}{ }^{u}}{z_{12}{ }^{2}} \frac{Q_{q}(1) Z_{\bar{q} g} K_{1}\left(Q_{q}(1) Z_{\bar{q} g}\right)}{x_{q}\left(1-x_{q}\right) Q^{2}+\vec{p}_{q}^{2}}\right.\right. \\
& \left.+\frac{\delta_{s \lambda_{q}} \delta_{-s_{g} \lambda_{\bar{q}}} x_{g} z_{12}{ }^{j}}{\left(x_{\bar{q}}+x_{g}\right)^{2}} \frac{z_{12}^{2}}{z_{12}}\left(z_{12}{ }^{u} x_{\bar{q}} K_{0}\left(Q_{q}(1) Z_{\bar{q} g}\right)+\frac{i p_{q}{ }^{u}}{Q_{q}(1)} Z_{\bar{q} g} K_{1}\left(Q_{q}(1) Z_{\bar{q} g}\right)\right)\right\} \\
& -\int_{0}^{1} d \alpha e^{i \frac{\alpha x_{\bar{q}}\left(\vec{p}_{q} \cdot \vec{z}_{12}\right)}{x_{\bar{q}}+x_{g}}-i \vec{p}_{q} \cdot \vec{z}_{1}}\left\{\left(x_{\bar{q}} \delta_{s \lambda_{\bar{q}}} \delta_{-s_{g} \lambda_{q}}+\left(\delta_{s \lambda_{q}}\left(x_{g} \delta_{-s_{g} \lambda_{\bar{q}}}+x_{q}\right)-x_{\bar{q}} \delta_{s \lambda_{\bar{q}}}\right)\right)\right. \\
& \times\left(\frac{x_{\bar{q}}}{x_{\bar{q}}+x_{g}} Q_{q}(\alpha) Z_{\bar{q} g q} K_{1}\left(Q_{q}(\alpha) Z_{\bar{q} g q}\right)\left(\frac{z_{12}{ }^{j} z_{12}{ }^{u}}{z_{12}{ }^{2}}+\frac{\alpha p_{q} p_{q}{ }^{u}}{x_{q}\left(1-x_{q}\right) Q^{2}+\alpha \vec{p}_{q}^{2}}\right)\right. \\
& \left.+\frac{K_{0}\left(Q_{q}(\alpha) Z_{\bar{q} g q}\right)}{x_{\bar{q}}+x_{g}}\left(\frac{i p_{q}{ }^{j} z_{12}{ }^{u} Z_{\bar{q} g q}{ }^{2}}{x_{q} z_{12}{ }^{2}}-\frac{z_{12}{ }^{j} i p_{q}{ }^{u} \alpha x_{\bar{q}}{ }^{2}}{x_{\bar{q}}+x_{g}}-x_{\bar{q}} \delta^{j u}\right)\right)+x_{\bar{q}} \delta_{s \lambda_{\bar{q}}} \delta_{-s_{g} \lambda_{q}} \\
& \times\left(-Q_{q}(\alpha) Z_{\bar{q} g q} K_{1}\left(Q_{q}(\alpha) Z_{\bar{q} g q}\right)\left(\frac{\alpha p_{q} p_{q}{ }^{u}}{x_{q}\left(1-x_{q}\right) Q^{2}+\alpha \vec{p}_{q}^{2}}+\frac{z_{12}{ }^{j} z_{12}{ }^{u}}{\vec{z}_{12}^{2}} \frac{(1-\alpha) x_{q} x_{\bar{q}}}{x_{g}+(1-\alpha) x_{q} x_{\bar{q}}}\right)\right. \\
& \left.\left.\left.+K_{0}\left(Q_{q}(\alpha) Z_{\bar{q} g q}\right)\left(\delta^{j u}+\alpha \frac{x_{\bar{q}} z_{12}{ }^{j} i p_{q}{ }^{u}}{x_{\bar{q}}+x_{g}}-(1-\alpha) \frac{x_{\bar{q}}}{x_{\bar{q}}+x_{g}} i p_{q}{ }^{j} z_{12}{ }^{u}\right)\right)\right\}\right] .
\end{aligned}
$$

Note that

$$
\int d \vec{z}_{3} F_{1}\left(p_{q}, p_{\bar{q}}, p_{g}, k, \vec{z}_{1}, \vec{z}_{3}, \vec{z}_{2}\right)^{\alpha} \varepsilon_{T \alpha}=-\left.\int d \vec{z}_{3} F_{1}\left(p_{q}, p_{\bar{q}}, p_{g}, k, \vec{z}_{3}, \vec{z}_{2}, \vec{z}_{1}\right)^{\alpha} \varepsilon_{T \alpha}\right|_{q \leftrightarrow \bar{q}},
$$

which allows one to evaluate the third integral involved in eq. (7.5).

For forward production $\vec{p}_{q}=\vec{p}_{\bar{q}}=\vec{p}_{g}=0$ one can simplify these expressions via eqs. (A.6) and (A.9), thus obtaining

$$
\begin{aligned}
& \int d \vec{z}_{3} F_{1}\left(p_{q}, p_{\bar{q}}, p_{g}, k, \vec{z}_{1}, \vec{z}_{2}, \vec{z}_{3}\right)^{\alpha} \varepsilon_{T \alpha} \\
& =2 i g \delta\left(k^{+}-p_{g}^{+}-p_{q}^{+}-p_{\bar{q}}^{+}\right) \theta\left(p_{g}^{+}-\sigma\right) \frac{\delta_{-\lambda_{\bar{q}} \lambda_{q}}}{x_{g} \sqrt{2 p_{g}^{+}}} \\
& \quad \times\left[\delta_{-s_{g} s} \frac{\left(\vec{z}_{12} \cdot \vec{\varepsilon}_{g}^{*}\right)\left(\vec{\varepsilon}_{T} \cdot \vec{z}_{12}\right)}{\vec{z}_{12}{ }^{2}}\left(K_{2}\left(Q Z_{q \bar{q}}\right)-2\left(1-x_{q}\right) K_{2}\left(Q Z_{122}\right)\right)\left(x_{q} \delta_{s \lambda_{q}}-x_{\bar{q}} \delta_{s \lambda_{\bar{q}}}\right)\right. \\
& \left.-\delta_{s s_{g}}\left(K_{0}\left(Q Z_{122}\right)\left(x_{q} x_{\bar{q}} \delta_{s \lambda_{q}}-\left(1-x_{q}\right)^{2} \delta_{s \lambda_{\bar{q}}}\right)+\frac{x_{\bar{q}} \delta_{s \lambda_{\bar{q}}}}{x_{\bar{q}}+x_{q}} K_{0}\left(Q Z_{q \bar{q}}\right)\right)\right]-(q \leftrightarrow \bar{q}),
\end{aligned}
$$


as well as

$$
\begin{aligned}
& \int d \vec{z}_{3} F_{1}\left(p_{q}, p_{\bar{q}}, p_{g}, k, \vec{z}_{3}, \vec{z}_{2}, \vec{z}_{1}\right)^{\alpha} \varepsilon_{T \alpha} \\
& =2 i g \delta\left(k^{+}-p_{g}^{+}-p_{q}^{+}-p_{\bar{q}}^{+}\right) \theta\left(p_{g}^{+}-\sigma\right) \frac{\delta_{-\lambda_{\bar{q}} \lambda_{q}}}{x_{q} \sqrt{2 p_{g}^{+}}} \\
& \quad \times\left[2 \delta_{-s_{g} s} \frac{\left(\vec{z}_{12} \cdot \vec{\varepsilon}_{g}^{*}\right)\left(\vec{\varepsilon}_{T} \cdot \vec{z}_{12}\right)}{\vec{z}_{12}{ }^{2}}\left(-\frac{x_{g}}{x_{\bar{q}}+x_{g}} K_{2}\left(Q Z_{\bar{q} g}\right)+\left(1-x_{\bar{q}}\right) K_{2}\left(Q Z_{121}\right)\right)\left(x_{q} \delta_{s \lambda_{q}}-x_{\bar{q}} \delta_{s \lambda_{\bar{q}}}\right)\right. \\
& \left.-\delta_{s s_{g}}\left(\delta_{s \lambda_{q}}\left(\frac{x_{g}}{x_{\bar{q}}+x_{g}}\right)^{2} K_{0}\left(Q Z_{\bar{q} g}\right)+\left(x_{q} x_{\bar{q}} \delta_{s \lambda_{\bar{q}}}-\left(1-x_{\bar{q}}\right)^{2} \delta_{s \lambda_{q}}\right) K_{0}\left(Q Z_{121}\right)\right)\right] .
\end{aligned}
$$

The expressions (7.10) and (7.16) can be used as a starting point for the description of e.g. meson production within the QCD collinear factorization, see ref. [45].

\section{Impact factor in the momentum space and in the linear approximation}

Here we will calculate the Fourier transform of the impact factors. We can rewrite the matrix element (4.2) as

$$
\begin{aligned}
M^{\alpha}= & \int d \vec{p}_{1} d \vec{p}_{2} d \vec{p}_{3} F_{1}\left(p_{q}, p_{\bar{q}}, p_{g}, z_{0}, \vec{p}_{1}, \vec{p}_{2}, \vec{p}_{3}\right)^{\alpha} \frac{1}{2}\left[N_{c}^{2}\left(\mathbf{U}_{32}+\mathbf{U}_{13}+\mathbf{U}_{32} \mathbf{U}_{13}\right)-\mathbf{U}_{12}\right]\left(\vec{p}_{1}, \vec{p}_{2}, \vec{p}_{3}\right) \\
& +\int d \vec{p}_{1} d \vec{p}_{2} \tilde{F}_{2}\left(p_{q}, p_{\bar{q}}, p_{g}, z_{0}, \vec{p}_{1}, \vec{p}_{2}\right)^{\alpha} \frac{N_{c}^{2}-1}{2} \mathbf{U}\left(\vec{p}_{1}, \vec{p}_{2}\right)
\end{aligned}
$$

Here the Fourier transforms are defined as

$$
\begin{aligned}
F_{1}\left(p_{q}, p_{\bar{q}}, p_{g}, z_{0}, \vec{p}_{1}, \vec{p}_{2}, \vec{p}_{3}\right)^{\alpha} & =\int \frac{d \vec{z}_{1}}{2 \pi} \frac{d \vec{z}_{2}}{2 \pi} \frac{d \vec{z}_{3}}{2 \pi} e^{i\left[\vec{p}_{1} \cdot \vec{z}_{1}+\vec{p}_{2} \cdot \vec{z}_{2}+\vec{p}_{3} \cdot \vec{z}_{3}\right]} F_{1}\left(p_{q}, p_{\bar{q}}, p_{g}, z_{0}, \vec{z}_{1}, \vec{z}_{2}, \vec{z}_{3}\right)^{\alpha}, \\
\tilde{F}_{2}\left(p_{q}, p_{\bar{q}}, p_{g}, z_{0}, \vec{p}_{1}, \vec{p}_{2}\right)^{\alpha} & =\int \frac{d \vec{z}_{1}}{2 \pi} \frac{d \vec{z}_{2}}{2 \pi} e^{i\left[\vec{p}_{1} \cdot \vec{z}_{1}+\vec{p}_{2} \cdot \vec{z}_{2}\right]} \tilde{F}_{2}\left(p_{q}, p_{\bar{q}}, p_{g}, z_{0}, \vec{z}_{1}, \vec{z}_{2}\right)^{\alpha}
\end{aligned}
$$

and

$$
\begin{aligned}
{\left[N_{c}^{2}\left(\mathbf{U}_{32}+\mathbf{U}_{13}+\mathbf{U}_{32} \mathbf{U}_{13}\right)-\mathbf{U}_{12}\right]\left(\vec{p}_{1}, \vec{p}_{2}, \vec{p}_{3}\right)=} & \int \frac{d \vec{z}_{1}}{2 \pi} \frac{d \vec{z}_{2}}{2 \pi} \frac{d \vec{z}_{3}}{2 \pi} e^{-i\left[\vec{p}_{1} \cdot \vec{z}_{1}+\vec{p}_{2} \cdot \vec{z}_{2}+\vec{p}_{3} \cdot \vec{z}_{3}\right]} \\
& \times\left[N_{c}^{2}\left(\mathbf{U}_{32}+\mathbf{U}_{13}+\mathbf{U}_{32} \mathbf{U}_{13}\right)-\mathbf{U}_{12}\right] \\
\mathbf{U}\left(\vec{p}_{1}, \vec{p}_{2}\right)= & \int \frac{d \overrightarrow{z_{1}}}{2 \pi} \frac{d \overrightarrow{z_{2}}}{2 \pi} e^{-i\left[\vec{p}_{1} \cdot \vec{z}_{1}+\vec{p}_{2} \cdot \vec{z}_{2}\right]} \mathbf{U}_{12}
\end{aligned}
$$

To get the linearized impact factor, one should neglect the term $\mathbf{U}_{32} \mathbf{U}_{13}$ and write

$$
\begin{aligned}
& {\left[N_{c}^{2}\left(\mathbf{U}_{32}+\mathbf{U}_{13}+\mathbf{U}_{32} \mathbf{U}_{13}\right)-\mathbf{U}_{12}\right]\left(\vec{p}_{1}, \vec{p}_{2}, \vec{p}_{3}\right)} \\
& \quad \sim 2 \pi\left[N_{c}^{2}\left(\delta\left(\vec{p}_{1}\right) \mathbf{U}\left(\vec{p}_{3}, \vec{p}_{2}\right)+\delta\left(\vec{p}_{2}\right) \mathbf{U}\left(\vec{p}_{1}, \vec{p}_{3}\right)\right)-\delta\left(\vec{p}_{3}\right) \mathbf{U}\left(\vec{p}_{1}, \vec{p}_{2}\right)\right] .
\end{aligned}
$$

Then for the matrix element $M^{\alpha}$ we get

$$
M^{\alpha}=\frac{1}{2} \int d \vec{p}_{1} d \vec{p}_{2} \mathbf{U}\left(\vec{p}_{1}, \vec{p}_{2}\right)\left\{\tilde{F}_{1}\left(p_{q}, p_{\bar{q}}, p_{g}, z_{0}, \vec{p}_{1}, \vec{p}_{2}\right)^{\alpha}+\left(N_{c}^{2}-1\right) \tilde{F}_{2}\left(p_{q}, p_{\bar{q}}, p_{g}, z_{0}, \vec{p}_{1}, \vec{p}_{2}\right)^{\alpha}\right\},
$$


where

$$
\begin{aligned}
\tilde{F}_{1}\left(p_{q}, p_{\bar{q}}, p_{g}, z_{0}, \vec{p}_{1}, \vec{p}_{2}\right)^{\alpha}= & 2 \pi N_{c}^{2} F_{1}\left(p_{q}, p_{\bar{q}}, p_{g}, z_{0}, 0, \vec{p}_{2}, \vec{p}_{1}\right)^{\alpha} \\
& +2 \pi N_{c}^{2} F_{1}\left(p_{q}, p_{\bar{q}}, p_{g}, z_{0}, \vec{p}_{1}, 0, \vec{p}_{2}\right)^{\alpha}-2 \pi F_{1}\left(p_{q}, p_{\bar{q}}, p_{g}, z_{0}, \vec{p}_{1}, \vec{p}_{2}, 0\right)^{\alpha} .
\end{aligned}
$$

Taking the Fourier transform of eq. (5.15) via eq. (B.1) from appendix B we get for the longitudinal photon

$$
\begin{aligned}
F_{1}\left(p_{q}, p_{\bar{q}}, p_{g}, z_{0}, \vec{p}_{1}, \vec{p}_{2}, \vec{p}_{3}\right)^{\alpha} \varepsilon_{L \alpha} \\
=\delta\left(k^{+}-p_{g}^{+}-p_{q}^{+}-p_{\bar{q}}^{+}\right) \delta\left(\vec{p}_{1 q}+\vec{p}_{2 \bar{q}}+\vec{p}_{3 g}\right) \theta\left(p_{g}^{+}-\sigma\right) \\
\quad \times \frac{\delta_{\lambda_{q},-\lambda_{\bar{q}}}}{\sqrt{2 p_{g}^{+}}} \frac{4 i Q g\left(x_{q}+x_{g} \delta_{-s_{g} \lambda_{\bar{q}}}\right)\left(\left(\vec{p}_{2 \bar{q}} \cdot \vec{\varepsilon}_{g}^{*}\right) x_{q}+\left(\vec{p}_{1 q} \cdot \vec{\varepsilon}_{g}^{*}\right)\left(1-x_{\bar{q}}\right)\right)}{\left(1-x_{\bar{q}}\right) x_{g} x_{q}\left(Q^{2}+\frac{\vec{p}_{2 \bar{q}^{2}}}{x_{\bar{q}}\left(1-x_{\bar{q}}\right)}\right)\left(Q^{2}+\frac{\vec{p}_{1 q}{ }^{2}}{x_{q}}+\frac{\vec{p}_{2 \bar{q}}{ }^{2}}{x_{\bar{q}}}+\frac{\vec{p}_{3 g}{ }^{2}}{x_{g}}\right)}-(q \leftrightarrow \bar{q}),
\end{aligned}
$$

where

$$
(q \leftrightarrow \bar{q}) \equiv\left(\lambda_{q}, x_{q}, \vec{p}_{1}, \vec{p}_{q} \leftrightarrow \lambda_{\bar{q}}, x_{\bar{q}}, \vec{p}_{2}, \vec{p}_{\bar{q}}\right) .
$$

One can check that this result is compatible with the wave function derived in ref. [49]. Using eq. (B.2), we get

$$
\begin{aligned}
\tilde{F}_{2}\left(p_{q}, p_{\bar{q}}, p_{g}, k, \vec{p}_{1}, \vec{p}_{2}\right)^{\alpha} \varepsilon_{L \alpha}= & \frac{4 i g Q}{\sqrt{2 p_{g}^{+}}} \theta\left(p_{g}^{+}-\sigma\right) \delta\left(k^{+}-p_{g}^{+}-p_{q}^{+}-p_{\bar{q}}^{+}\right) \delta\left(\vec{p}_{1 q}+\vec{p}_{2 \bar{q}}-\vec{p}_{g}\right) \\
& \times \delta_{\lambda_{q},-\lambda_{\bar{q}}} \frac{x_{\bar{q}}+\delta_{-s_{g} \lambda_{q}} x_{g}}{x_{\bar{q}} x_{g}} \frac{2 \pi}{Q^{2}+\frac{\vec{p}_{1 q}^{2}}{x_{q}\left(1-x_{q}\right)}} \frac{\vec{P}_{\bar{q}} \cdot \vec{\varepsilon}_{g}^{*}}{\vec{P}_{\bar{q}}^{2}}-(q \leftrightarrow \bar{q}) .
\end{aligned}
$$

For the transverse photon, using eqs. (B.3) and (B.4) we have

$$
\begin{aligned}
& F_{1}\left(p_{q}, p_{\bar{q}}, p_{g}, z_{0}, \vec{p}_{1}, \vec{p}_{2}, \vec{p}_{3}\right)^{\alpha} \varepsilon_{T \alpha} \\
& =\frac{-4 i g}{\sqrt{2 p_{g}^{+}}} \delta\left(k^{+}-p_{g}^{+}-p_{q}^{+}-p_{\bar{q}}^{+}\right) \delta\left(\vec{p}_{1 q}+\vec{p}_{2 \bar{q}}+\vec{p}_{3 g}\right) \theta\left(p_{g}^{+}-\sigma\right) \\
& \times \delta_{\lambda_{q},-\lambda_{\bar{q}}} \frac{\left(\vec{p}_{2 \bar{q}} \cdot \vec{\varepsilon}_{g}^{*}\right)\left(x_{\bar{q}}+x_{g}\right)+\left(\vec{p}_{1 q} \cdot \vec{\varepsilon}_{g}^{*}\right) x_{\bar{q}}}{\left(\vec{p}_{2 \bar{q}}\left(x_{\bar{q}}+x_{g}\right)+\vec{p}_{1 q} x_{\bar{q}}\right)^{2}}\left\{\frac{\left(x_{q}-\delta_{s \lambda_{\bar{q}}}\right)\left(x_{\bar{q}}+x_{g} \delta_{-s_{g} \lambda_{q}}\right)}{x_{q}}\right. \\
& \times\left(\frac{\left(\vec{p}_{1 q} \cdot \vec{\varepsilon}_{T}\right)\left(x_{g}+x_{q}\right)+\left(\vec{p}_{2 \bar{q}} \cdot \vec{\varepsilon}_{T}\right) x_{q}}{x_{g}\left(\frac{\vec{p}_{1 q}{ }^{2}}{x_{q}}+\frac{\vec{p}_{2 q}{ }^{2}}{x_{\bar{q}}}+\frac{\vec{p}_{3 g}{ }^{2}}{x_{g}}+Q^{2}\right)}-\frac{\left(\vec{p}_{1 q} \cdot \vec{\varepsilon}_{T}\right)}{\left(x_{\bar{q}}+x_{g}\right)\left(\frac{\vec{p}_{1 q}{ }^{2}}{x_{q}\left(x_{\bar{q}}+x_{g}\right)}+Q^{2}\right)}\right) \\
& \left.+\left(x_{\bar{q}}+x_{g} \delta_{-s_{g} \lambda_{q}}-\delta_{s \lambda_{q}}\right) \frac{\left(\vec{p}_{2 \bar{q}} \cdot \vec{\varepsilon}_{T}\right)\left(x_{\bar{q}}+x_{g}\right)+\left(\vec{p}_{1 q} \cdot \vec{\varepsilon}_{T}\right) x_{\bar{q}}}{x_{g}\left(\frac{\vec{p}_{q^{2}}{ }^{2}}{x_{q}}+\frac{\vec{p}_{2 \bar{q}}{ }^{2}}{x_{\bar{q}}}+\frac{\vec{p}_{3 g^{2}}}{x_{g}}+Q^{2}\right)}\right\}-(q \leftrightarrow \bar{q}) \\
& =\frac{2 i g}{\sqrt{2 p_{g}^{+}}} \frac{\delta\left(k^{+}-p_{g}^{+}-p_{q}^{+}-p_{\bar{q}}^{+}\right) \delta\left(\vec{p}_{1 q}+\vec{p}_{2 \bar{q}}+\vec{p}_{3 g}\right) \theta\left(p_{g}^{+}-\sigma\right) \delta_{-\lambda_{\bar{q}} \lambda_{q}}}{Q^{2}\left(1-x_{q}\right)\left(\frac{\vec{p}_{1 q}^{2}}{x_{q}}+\frac{\vec{p}_{\bar{q}}^{2}}{x_{\bar{q}}}+\frac{\vec{p}_{3 g^{2}}}{x_{g}}+Q^{2}\right)}\left\{\delta_{s s_{g}} \delta_{s \lambda_{q}}\right. \\
& \left.+2\left(\vec{p}_{1 q} \cdot \vec{\varepsilon}_{T}\right)\left(\left(\vec{p}_{2 \bar{q}} \cdot \vec{\varepsilon}_{g}^{*}\right)\left(x_{\bar{q}}+x_{g}\right)+\left(\vec{p}_{1 q} \cdot \vec{\varepsilon}_{g}^{*}\right) x_{\bar{q}}\right) \frac{\left(x_{q}-\delta_{s \lambda_{\bar{q}}}\right)\left(x_{g} \delta_{-s_{g} \lambda_{q}}+x_{\bar{q}}\right)}{\left(1-x_{q}\right) x_{q} x_{\bar{q}} x_{g}\left(Q^{2}+\frac{\vec{p}_{1 q}^{2}}{\left(1-x_{q}\right) x_{q}}\right)}\right\}-(q \leftrightarrow \bar{q}) .
\end{aligned}
$$


Again, one can check that this result is compatible with the wave function derived in ref. [49]. Finally, using eq. (B.5) we find

$$
\begin{aligned}
& \tilde{F}_{2}\left(p_{q}, p_{\bar{q}}, p_{g}, k, \vec{p}_{1}, \vec{p}_{2}\right)^{\alpha} \varepsilon_{T \alpha} \\
& =-4 g \theta\left(p_{g}^{+}-\sigma\right) \delta\left(k^{+}-p_{g}^{+}-p_{q}^{+}-p_{\bar{q}}^{+}\right) \delta\left(\vec{p}_{1 q}+\vec{p}_{2 \bar{q}}-\vec{p}_{g}\right) \frac{\delta_{\lambda_{q},-\lambda_{\bar{q}}}}{\sqrt{2 p_{g}^{+}}} \\
& \quad \times \frac{\left(\delta_{\lambda_{\bar{q}} s}-x_{q}\right)\left(\delta_{-s_{g} \lambda_{q}} x_{g}+x_{\bar{q}}\right)}{x_{\bar{q}} x_{g}} \frac{2 \pi i\left(\vec{p}_{1 q} \cdot \vec{\varepsilon}_{T}\right)}{x_{q}\left(1-x_{q}\right) Q^{2}+\vec{p}_{1 q}^{2}} \frac{\vec{P}_{\bar{q}} \cdot \vec{\varepsilon}_{g}^{*}}{\vec{P}_{\bar{q}}^{2}}-(q \leftrightarrow \bar{q}) .
\end{aligned}
$$

The formulas in the momentum space derived in this section constitute a convenient starting point for calculations of phenomenologically important observables such as cross sections etc.

\section{Conclusions}

Based on the QCD shock-wave approach [28, 36, 37], we rederived the $\gamma^{*} \rightarrow q \bar{q}$ impact factor. Using the same approach, we computed the general expression for the $\gamma^{*} \rightarrow q \bar{q} g$ impact factor for the first time, without collinear of soft approximations. The contribution of the diagrams with the gluons crossing the shock-wave, calculated using Balitsky's formalism, are consistent with the results for the $\gamma^{*} \rightarrow q \bar{q} g$ wave function obtained in ref. [49], based on old-fashioned perturbation theory.

The results we obtained, in coordinate space, are very suitable for phenomenological studies of diffractive processes since they allow for the implementation of saturation models, when considering the color-singlet channel. The measurement of dijet production in DDIS was recently performed [50], and a precise comparison of dijet versus triple-jet production, which has not been performed yet at HERA [51], would be very useful to get a deeper understanding of the QCD mechanism underlying diffraction. Such a ratio would provide an observable possibly more independent of any saturation effect. A quantitative, first principle analysis of this would require an evaluation of virtual corrections to the $\gamma^{*} \rightarrow q \bar{q}$ impact factor, which are left for further studies.

Our results could also be relevant for photo-production of diffractive jets [52, 53], the hard scale being provided by the invariant mass of the produced state. Indeed, usual collinear descriptions of this process rely on a resolved Pomeron contribution, which is the sum of a direct interaction of the photon with quarks or gluons originating from the pomeron, and a resolved photon-pomeron interaction. In the region $x_{\gamma} \sim 1$ ( $x_{\gamma}$ is the longitudinal momentum fraction carried by the partons coming from the photon), an additional direct coupling of a Pomeron to the impact factor could be important, in view of the collinear factorization breaking of the above described picture which has been the matter of discussions [54, 55]. Since our results are expressed in terms of a shock-wave factor, they can be used both for inclusive (considering the color octet in the $t$-channel, by modifying formula (4.2) and diffractive (in the color-singlet case) jet production, the ratio of amplitudes providing an interesting observable to evaluate gap survival probabilities [54-56]. 
Furthermore, our results, expressed in terms of a shock-wave factor, are a natural starting point for studies of higher-twist effects, which could be investigated by an appropriate expansion of $U$ operators in powers of the coupling, in order to study the effect of multigluon exchange in the $t$-channel.

Finally, diffractive open charm production was measured at HERA [57] and studied in the large $M$ limit based on the direct coupling between a Pomeron and a $q \bar{q}$ or a $q \bar{q} g$ state, with massive quarks [22]. The extension of our result to the case of massive quark, is left for future analysis.

Our result is therefore a first step for phenomenological studies of diffraction, which could be of relevance in future $e-p$ and $e-A$ colliders, like EIC and LHeC, as well as for ultraperipheral processes, which could be studied at LHC.

\section{Acknowledgments}

We would like to thank A. Besse, G. Beuf, L. Motyka, Al Mueller, S. Munier and M. Sadzikowski for discussions. A.V.G. thanks V.S. Fadin and A.V. Reznichenko for helpful discussions and the LPT Orsay and NCBJ in Warsaw for hospitality while part of this work was being done. A.V.G. also acknowledges support of president grant MK-525.2013.2 and RFBR grant 13-02-01023. This work was partially supported by the PEPS-PTI PHENODIFF, the PRC0731 DIFF-QCD, the Polish Grant NCN No. DEC-2011/01/B/ST2/03915, the COPIN-IN2P3 Agreement and the Joint Research Activity Study of Strongly Interacting Matter (acronym HadronPhysics3, Grant Agreement n.283286) under the Seventh Framework Programme of the European Community.

\section{A Integrals necessary for linearization}

We need the following integrals

$$
\left.\int d \vec{z}_{1} e^{-i \vec{p}_{q} \cdot \vec{z}_{1}} K_{0}\left(Q Z_{123}\right)\right|_{\vec{z}_{3} \rightarrow \vec{z}_{1}}=\frac{2 \pi}{x_{q}\left(1-x_{q}\right)} e^{-i \frac{x_{\bar{q}}\left(\vec{p}_{q} \cdot \vec{z}_{2}\right)+x_{g}\left(\vec{p}_{q} \cdot \vec{z}_{1}\right)}{x_{g}+x_{\bar{q}}}} \frac{Z_{\bar{q} g} K_{1}\left(Z_{\bar{q} g} Q_{q}(1)\right)}{Q_{q}(1)}
$$

where we define

$$
\begin{aligned}
& Z_{i j}=\sqrt{\frac{x_{i} x_{j}}{x_{i}+x_{j}} \vec{z}_{12}^{2}}, \quad Q_{i}(\alpha)=\sqrt{\frac{\alpha \vec{p}_{i}^{2}}{\left(1-x_{i}\right) x_{i}}+Q^{2}} . \\
& \int d \vec{z}_{3} e^{-i \vec{p}_{g} \cdot \vec{z}_{3}} \frac{\vec{z}_{32}}{\vec{z}_{32}^{2}} K_{0}\left(Q Z_{123}\right) \\
&=-\pi i e^{-i \vec{p}_{g} \cdot \vec{z}_{2}} \int_{-\infty}^{0} d t e^{\frac{i t x_{g} x_{q} Q^{2} \vec{z}_{21}^{2}}{2}-i \frac{\left(x_{\bar{q}}+x_{g}\right)+i 0}{2 t x_{g}}} \frac{t \vec{p}_{g}+\frac{\vec{z}_{21}}{\vec{z}_{21}^{2}}}{\left(t \vec{p}_{g}+\frac{\vec{z}_{21}}{\vec{z}_{21}^{2}}\right)^{2}}\left(e^{\frac{i x_{q} \vec{z}_{21}^{2}}{2\left(x_{\bar{q}}+x_{q}\right) t}\left(t \vec{p}_{g}+\frac{\vec{z}_{21}}{\vec{z}_{21}^{2}}\right)^{2}}-1\right) \\
&=-\frac{\pi e^{-i \vec{p}_{g} \cdot \vec{z}_{2}}}{\left(1-x_{g}\right) x_{g}} \int_{0}^{1} d \alpha e^{\alpha \frac{i x_{q}\left(\vec{z}_{21} \cdot \vec{p} g\right)}{x_{\bar{q}}+x_{q}}}\left(\frac{i \vec{p}_{g} Z_{q \bar{q} g}}{Q_{g}(\alpha)} K_{1}\left(Q_{g}(\alpha) Z_{q \bar{q} g}\right)+x_{g} x_{q} \vec{z}_{21} K_{0}\left(Q_{g}(\alpha) Z_{q \bar{q} g}\right)\right),
\end{aligned}
$$


where

$$
Z_{q \bar{q} g}=\sqrt{x_{q} \frac{x_{\bar{q}}+(1-\alpha) x_{q} x_{g}}{x_{\bar{q}}+x_{q}} \vec{z}_{21}^{2}} .
$$

To get the previous result, we used the following parametrization

$$
\begin{aligned}
\int d \vec{r} \frac{\vec{r}}{\vec{r} 2} e^{i\left(a \vec{r}^{2}+b(\vec{r}+\vec{\rho})^{2}\right)-i \vec{p} \cdot \vec{r}} & =-2 \pi \frac{i(2 b \vec{\rho}-\vec{p})}{(2 b \vec{\rho}-\vec{p})^{2}} e^{i b \vec{\rho}^{2}}\left(e^{-\frac{i(2 b \vec{\rho}-\vec{p})^{2}}{4(a+b)}}-1\right) \\
& =-\frac{\pi}{2(a+b)}(2 b \vec{\rho}-\vec{p}) e^{i b \vec{\rho}^{2}} \int_{0}^{1} d \alpha e^{-\alpha \frac{i(2 b \vec{\rho}-\vec{p})^{2}}{4(a+b)}}
\end{aligned}
$$

In the simpler case $\vec{p}_{q}=0$ the integral can be fully reduced to

$$
\int d \vec{z}_{3} \frac{\vec{z}_{32}}{\vec{z}_{32}^{2}} K_{0}\left(Q Z_{123}\right)=-\frac{2 \pi}{x_{g} x_{q} Q} \frac{\vec{z}_{21}}{\vec{z}_{21}^{2}}\left(Z_{q \bar{q}} K_{1}\left(Q Z_{q \bar{q}}\right)-Z_{122} K_{1}\left(Q Z_{122}\right)\right)
$$

where

$$
Z_{122}=\sqrt{x_{q}\left(x_{\bar{q}}+x_{g}\right) \vec{z}_{21}^{2}}
$$

Using the integral (A.5), we also get

$$
\begin{aligned}
& \int d \vec{z}_{3} e^{-i \vec{p}_{g} \cdot \vec{z}_{3}} \frac{z_{32}^{j}}{\vec{z}_{32}^{2}} \frac{\partial}{\partial z_{3}^{l}} K_{0}\left(Q Z_{123}\right) \\
& =-\pi e^{-i \vec{p}_{g} \cdot \vec{z}_{2}} \int_{0}^{1} d \alpha e^{\alpha \frac{i x_{q}\left(\vec{z}_{21} \cdot \vec{p}_{g}\right)}{x_{q}+x_{\bar{q}}}}\left[\left(\delta^{j l}+i p_{g}^{j} z_{12}^{l} \frac{x_{q}}{x_{\bar{q}}+x_{q}}\right)\right. \\
& \quad \times K_{0}\left(Q_{g}(\alpha) Z_{q \bar{q} g}\right)-\frac{x_{q}^{2} x_{g}}{x_{\bar{q}}+x_{q}} z_{12}^{l} z_{12}^{j} \frac{Q_{g}(\alpha)}{Z_{q \bar{q} g}} K_{1}\left(Q_{g}(\alpha) Z_{q \bar{q} g}\right)(1-\alpha) \\
& \left.\quad-\frac{x_{q}\left(i p_{g}^{l} z_{12}^{j}+i p_{g}^{j} z_{12}^{l}\right)}{x_{q}+x_{\bar{q}}} \alpha K_{0}\left(Q_{g}(\alpha) Z_{q \bar{q} g}\right)+\frac{i p_{g}^{l} i p_{g}^{j}}{x_{g}\left(x_{q}+x_{\bar{q}}\right)} \alpha \frac{Z_{q \bar{q} g}}{Q_{g}(\alpha)} K_{1}\left(Q_{g}(\alpha) Z_{q \bar{q} g}\right)\right] .
\end{aligned}
$$

As before, in the simpler case $\vec{p}_{g}=0$ the integral can be fully reduced to

$$
\begin{aligned}
\int d \vec{z}_{3} \frac{z_{31}^{j}}{\vec{z}_{31}^{2}} \frac{\partial}{\partial z_{3}^{l}} K_{0}\left(Q Z_{123}\right)= & -\frac{2 \pi x_{q}}{x_{\bar{q}} x_{g}}\left(\delta^{j l}-2 \frac{z_{12}^{l} z_{12}^{j}}{\vec{z}_{21}^{2}}\right)\left(\frac{K_{1}\left(Q Z_{q \bar{q}}\right)}{Q Z_{q \bar{q}}}-\frac{K_{1}\left(Q Z_{121}\right)}{Q Z_{121}}\right) \\
& +2 \pi\left(\delta^{j l} \frac{K_{1}\left(Q Z_{121}\right)}{Q Z_{121}}-\frac{z_{12}^{l} z_{12}^{j}}{\vec{z}_{21}^{2}} K_{2}\left(Q Z_{121}\right)\right) .
\end{aligned}
$$

The integrals (A.3) and (A.8) are convergent and can be evaluated numerically. 


\section{B Integrals necessary for Fourier transform}

We here provide the set of Fourier transforms of modified Bessel functions we used in our calculation.

$$
\begin{aligned}
& \int \frac{d \vec{z}_{1}}{2 \pi} \frac{d \vec{z}_{2}}{2 \pi} e^{i\left[\vec{q}_{1} \cdot \vec{z}_{1}+\vec{q}_{2} \cdot \vec{z}_{2}\right]} \frac{z_{1}^{j}}{\vec{z}_{1}^{2}} K_{0}\left(Q \sqrt{x_{g} x_{q} \vec{z}_{1}^{2}+x_{q} x_{\bar{q}} \vec{z}_{21}^{2}+x_{\bar{q}} x_{g} \vec{z}_{2}^{2}}\right) \\
& =\frac{i\left(q_{2}^{j} x_{q}+q_{1}^{j}\left(1-x_{\bar{q}}\right)\right)}{\left(1-x_{\bar{q}}\right) x_{\bar{q}} x_{g} x_{q}\left(Q^{2}+\frac{\vec{q}_{2}^{2}}{x_{\bar{q}}\left(1-x_{\bar{q}}\right)}\right)\left(Q^{2}+\frac{\vec{q}_{1}^{2}}{x_{q}}+\frac{{\overrightarrow{q_{2}}}^{2}}{x_{\bar{q}}}+\frac{\left(\vec{q}_{1}+\vec{q}_{2}\right)^{2}}{x_{g}}\right)} . \\
& \int \frac{d \vec{z}_{1}}{2 \pi} e^{i \vec{q}_{1} \cdot \vec{z}_{1}} K_{0}\left(Q \sqrt{x_{q}\left(x_{g}+x_{\bar{q}}\right) \vec{z}_{1}^{2}}\right)=\frac{1}{\left(1-x_{q}\right) x_{q} Q^{2}+{\overrightarrow{q_{1}}}^{2}} . \\
& \int \frac{d \vec{z}_{1}}{2 \pi} \frac{d \vec{z}_{2}}{2 \pi} e^{i\left[\vec{q}_{1} \cdot \vec{z}_{1}+\vec{q}_{2} \cdot \vec{z}_{2}\right]} \frac{z_{1}^{j} z_{2}^{\beta}}{\vec{z}_{2}^{2}} \frac{K_{1}\left(Q \sqrt{x_{g} x_{q} \vec{z}_{1}^{2}+x_{q} x_{\bar{q}} \vec{z}_{21}^{2}+x_{\bar{q}} x_{g} \vec{z}_{2}^{2}}\right)}{\sqrt{x_{g} x_{q} \vec{z}_{1}^{2}+x_{q} x_{\bar{q}} \vec{z}_{21}^{2}+x_{\bar{q}} x_{g} \vec{z}_{2}^{2}}} \\
& =\frac{x_{\bar{q}}\left(\delta^{j \beta}-\frac{2\left(q_{2}^{j}\left(x_{\bar{q}}+x_{g}\right)+q_{1} x_{\bar{q}}\right)\left(q_{2}{ }^{\beta}\left(x_{\bar{q}}+x_{g}\right)+q_{1}{ }^{\beta} x_{\bar{q}}\right)}{\left(\vec{q}_{2}\left(x_{\bar{q}}+x_{g}\right)+\vec{q}_{1} x_{\bar{q}}\right)^{2}}\right)}{2 Q x_{q}\left(\vec{q}_{2}\left(x_{\bar{q}}+x_{g}\right)+\overrightarrow{q_{1}} x_{\bar{q}}\right)^{2}} \ln \left(\frac{\frac{q_{2}{ }^{2}}{x_{\bar{q}}}+\frac{\left(q_{1}+q_{2}\right)^{2}}{x_{g}}+\frac{q_{1}{ }^{2}}{x_{q}}+Q^{2}}{\frac{q_{1}{ }^{2}}{x_{q}\left(1-x_{q}\right)}+Q^{2}}\right) \\
& +\frac{q_{2}{ }^{\beta}\left(x_{\bar{q}}+x_{g}\right)+q_{1}{ }^{\beta} x_{\bar{q}}}{Q x_{q}^{2}\left(\vec{q}_{2}\left(x_{\bar{q}}+x_{g}\right)+\vec{q}_{1} x_{\bar{q}}\right)^{2}}\left(\frac{q_{1}{ }^{j}\left(x_{g}+x_{q}\right)+q_{2}{ }^{j} x_{q}}{x_{g}\left(\frac{q_{2}{ }^{2}}{x_{\bar{q}}}+\frac{\left(q_{1}+q_{2}\right)^{2}}{x_{g}}+\frac{q_{1}{ }^{2}}{x_{q}}+Q^{2}\right)}-\frac{q_{1}{ }^{j}}{\left(x_{\bar{q}}+x_{g}\right)\left(\frac{q_{1}{ }^{2}}{x_{q}\left(x_{\bar{q}}+x_{g}\right)}+Q^{2}\right)}\right) . \\
& \int \frac{d \vec{z}_{1}}{2 \pi} \frac{d \vec{z}_{2}}{2 \pi} e^{i\left[\vec{q}_{1} \cdot \vec{z}_{1}+\vec{q}_{2} \cdot \vec{z}_{2}\right]} \frac{z_{2}^{j} z_{2}^{\beta}}{\vec{z}_{2}^{2}} \frac{K_{1}\left(Q \sqrt{x_{g} x_{q} \vec{z}_{1}^{2}+x_{q} x_{\bar{q}} \vec{z}_{21}^{2}+x_{\bar{q}} x_{g} \vec{z}_{2}^{2}}\right)}{\sqrt{x_{g} x_{q} \vec{z}_{1}^{2}+x_{q} x_{\bar{q}} \vec{z}_{21}^{2}+x_{\bar{q}} x_{g} \vec{z}_{2}^{2}}} \\
& =\frac{\left(x_{\bar{q}}+x_{g}\right)\left(\delta^{j \beta}-\frac{2\left(q_{2}^{j}\left(x_{\bar{q}}+x_{g}\right)+q_{1}{ }^{j} x_{\bar{q}}\right)\left(q_{2}{ }^{\beta}\left(x_{\bar{q}}+x_{g}\right)+q_{1}{ }^{\beta} x_{\bar{q}}\right)}{\left(\vec{q}_{2}\left(x_{\bar{q}}+x_{g}\right)+\vec{q}_{1} x_{\bar{q}}\right)^{2}}\right)}{2 Q x_{q}\left(\vec{q}_{2}\left(x_{\bar{q}}+x_{g}\right)+\vec{q}_{1} x_{\bar{q}}\right)^{2}} \ln \left(\frac{\frac{q_{2}^{2}}{x_{\bar{q}}}+\frac{\left(q_{1}+q_{2}\right)^{2}}{x_{g}}+\frac{q_{1}^{2}}{x_{q}}+Q^{2}}{\frac{q_{1}^{2}}{x_{q}\left(1-x_{q}\right)}+Q^{2}}\right) \\
& +\frac{\left(q_{2}^{j}\left(x_{\bar{q}}+x_{g}\right)+q_{1}^{j} x_{\bar{q}}\right)\left(q_{2}{ }^{\beta}\left(x_{\bar{q}}+x_{g}\right)+q_{1}{ }^{\beta} x_{\bar{q}}\right)}{Q x_{g} x_{q} x_{\bar{q}}\left(\vec{q}_{2}\left(x_{\bar{q}}+x_{g}\right)+\vec{q}_{1} x_{\bar{q}}\right)^{2}\left(\frac{q_{2}^{2}}{x_{\bar{q}}}+\frac{\left(q_{1}+q_{2}\right)^{2}}{x_{g}}+\frac{q_{1}^{2}}{x_{q}}+Q^{2}\right)} . \\
& \int \frac{d \vec{z}_{1}}{2 \pi} e^{i \vec{q}_{1} \vec{z}_{1}} z_{1}^{j} \frac{K_{1}\left(Q \sqrt{x_{q}\left(x_{g}+x_{\bar{q}}\right) \vec{z}_{1}^{2}}\right)}{\sqrt{x_{q}\left(x_{g}+x_{\bar{q}}\right) \vec{z}_{1}^{2}}}=\frac{i q_{1}^{j}}{\left(1-x_{q}\right) x_{q} Q\left(\left(1-x_{q}\right) x_{q} Q^{2}+\vec{q}_{1}^{2}\right)} .
\end{aligned}
$$

Open Access. This article is distributed under the terms of the Creative Commons Attribution License (CC-BY 4.0), which permits any use, distribution and reproduction in any medium, provided the original author(s) and source are credited.

\section{References}

[1] M. Wusthoff and A.D. Martin, The QCD description of diffractive processes, J. Phys. G 25 (1999) R309 [hep-ph/9909362] [INSPIRE].

[2] G. Wolf, Review of High Energy Diffraction in Real and Virtual Photon Proton scattering at HERA, Rept. Prog. Phys. 73 (2010) 116202 [arXiv:0907.1217] [InSPIRE].

[3] H1 collaboration, A. Aktas et al., Diffractive deep-inelastic scattering with a leading proton at HERA, Eur. Phys. J. C 48 (2006) 749 [hep-ex/0606003] [InSPIRE]. 
[4] H1 collaboration, A. Aktas et al., Measurement and QCD analysis of the diffractive deep-inelastic scattering cross-section at HERA, Eur. Phys. J. C 48 (2006) 715 [hep-ex/0606004] [INSPIRE].

[5] ZEUS collaboration, S. Chekanov et al., Dissociation of virtual photons in events with a leading proton at HERA, Eur. Phys. J. C 38 (2004) 43 [hep-ex/0408009] [INSPIRE].

[6] ZEUS collaboration, S. Chekanov et al., Study of deep inelastic inclusive and diffractive scattering with the ZEUS forward plug calorimeter, Nucl. Phys. B 713 (2005) 3 [hep-ex/0501060] [INSPIRE].

[7] F.D. Aaron et al., Measurement of the cross section for diffractive deep-inelastic scattering with a leading proton at HERA, Eur. Phys. J. C 71 (2011) 1578 [arXiv:1010.1476] [INSPIRE].

[8] H1 collaboration, F.D. Aaron et al., Inclusive Measurement of Diffractive Deep-Inelastic Scattering at HERA, Eur. Phys. J. C 72 (2012) 2074 [arXiv: 1203.4495] [INSPIRE].

[9] ZEUS collaboration, S. Chekanov et al., Deep inelastic scattering with leading protons or large rapidity gaps at HERA, Nucl. Phys. B 816 (2009) 1 [arXiv: 0812. 2003] [INSPIRE].

[10] H1, ZEUS collaborations, F.D. Aaron et al., Combined inclusive diffractive cross sections measured with forward proton spectrometers in deep inelastic ep scattering at HERA, Eur. Phys. J. C 72 (2012) 2175 [arXiv:1207.4864] [INSPIRE].

[11] J.C. Collins, Proof of factorization for diffractive hard scattering, Phys. Rev. D 57 (1998) 3051 [Erratum ibid. D 61 (2000) 019902] [hep-ph/9709499] [INSPIRE].

[12] J. Bartels, J.R. Ellis, H. Kowalski and M. Wusthoff, An analysis of diffraction in deep inelastic scattering, Eur. Phys. J. C 7 (1999) 443 [hep-ph/9803497] [INSPIRE].

[13] A.D. Martin, M.G. Ryskin and G. Watt, Diffractive parton distributions from perturbative QCD, Eur. Phys. J. C 44 (2005) 69 [hep-ph/0504132] [INSPIRE].

[14] A.D. Martin, M.G. Ryskin and G. Watt, Diffractive parton distributions from H1 data, Phys. Lett. B 644 (2007) 131 [hep-ph/0609273] [INSPIRE].

[15] M. Wusthoff, Photon diffractive dissociation in deep inelastic scattering, DESY-95-166 [INSPIRE].

[16] M. Wusthoff, Large rapidity gap events in deep inelastic scattering, Phys. Rev. D 56 (1997) 4311 [hep-ph/9702201] [InSPIRE].

[17] V.N. Gribov and L.N. Lipatov, Deep inelastic e p scattering in perturbation theory, Sov. J. Nucl. Phys. 15 (1972) 438 [inSPIRE].

[18] L.N. Lipatov, The parton model and perturbation theory, Sov. J. Nucl. Phys. 20 (1975) 94 [INSPIRE].

[19] G. Altarelli and G. Parisi, Asymptotic Freedom in Parton Language, Nucl. Phys. B 126 (1977) 298 [inSPIRE].

[20] Y.L. Dokshitzer, Calculation of the Structure Functions for Deep Inelastic Scattering and $e^{+} e^{-}$Annihilation by Perturbation Theory in Quantum Chromodynamics., Sov. Phys. JETP 46 (1977) 641 [INSPIRE].

[21] J. Bartels, H. Jung and M. Wusthoff, Quark-antiquark-gluon jets in DIS diffractive dissociation, Eur. Phys. J. C 11 (1999) 111 [hep-ph/9903265] [INSPIRE]. 
[22] J. Bartels, H. Jung and A. Kyrieleis, Massive cēg: Calculation in diffractive DIS and diffractive $D^{*}$ production at HERA, Eur. Phys. J. C 24 (2002) 555 [hep-ph/0204269] [INSPIRE].

[23] V.S. Fadin, E.A. Kuraev and L.N. Lipatov, On the Pomeranchuk Singularity in Asymptotically Free Theories, Phys. Lett. B 60 (1975) 50 [INSPIRE].

[24] E.A. Kuraev, L.N. Lipatov and V.S. Fadin, Multi-Reggeon Processes in the Yang-Mills Theory, Sov. Phys. JETP 44 (1976) 443 [Erratum ibid. 45 (1977) 199] [InSPIRE].

[25] E.A. Kuraev, L.N. Lipatov and V.S. Fadin, The Pomeranchuk Singularity in Nonabelian Gauge Theories, Sov. Phys. JETP 45 (1977) 199 [INSPIRE].

[26] I.I. Balitsky and L.N. Lipatov, The Pomeranchuk Singularity in Quantum Chromodynamics, Sov. J. Nucl. Phys. 28 (1978) 822 [inSPIRE].

[27] C. Marquet, A unified description of diffractive deep inelastic scattering with saturation, Phys. Rev. D 76 (2007) 094017 [arXiv:0706.2682] [INSPIRE].

[28] I. Balitsky, Operator expansion for high-energy scattering, Nucl. Phys. B 463 (1996) 99 [hep-ph/9509348] [INSPIRE].

[29] I. Balitsky, Factorization for high-energy scattering, Phys. Rev. Lett. 81 (1998) 2024 [hep-ph/9807434] [INSPIRE].

[30] I. Balitsky, Factorization and high-energy effective action, Phys. Rev. D 60 (1999) 014020 [hep-ph/9812311] [INSPIRE].

[31] I. Balitsky, Effective field theory for the small-x evolution, Phys. Lett. B 518 (2001) 235 [hep-ph/0105334] [INSPIRE].

[32] L. Motyka, M. Sadzikowski and W. Slominski, Evidence of strong higher twist effects in diffractive DIS at HERA at moderate $Q^{2}$, Phys. Rev. D 86 (2012) 111501 [arXiv: 1203.5461] [INSPIRE].

[33] I.I. Balitsky and V.M. Braun, Evolution Equations for QCD String Operators, Nucl. Phys. B 311 (1989) 541 [INSPIRE].

[34] Y.V. Kovchegov, Small-x $F_{2}$ structure function of a nucleus including multiple Pomeron exchanges, Phys. Rev. D 60 (1999) 034008 [hep-ph/9901281] [INSPIRE].

[35] Y.V. Kovchegov, Unitarization of the BFKL Pomeron on a nucleus, Phys. Rev. D 61 (2000) 074018 [hep-ph/9905214] [InSPIRE].

[36] I. Balitsky and G.A. Chirilli, Photon impact factor in the next-to-leading order, Phys. Rev. D 83 (2011) 031502 [arXiv: 1009.4729] [InSPIRE].

[37] I. Balitsky and G.A. Chirilli, Photon impact factor and $k_{T}$-factorization for DIS in the next-to-leading order, Phys. Rev. D 87 (2013) 014013 [arXiv: 1207. 3844] [INSPIRE].

[38] G.A. Chirilli, B.-W. Xiao and F. Yuan, One-loop Factorization for Inclusive Hadron Production in $p A$ Collisions in the Saturation Formalism, Phys. Rev. Lett. 108 (2012) 122301 [arXiv:1112.1061] [InSPIRE].

[39] G.A. Chirilli, B.-W. Xiao and F. Yuan, Inclusive Hadron Productions in pA Collisions, Phys. Rev. D 86 (2012) 054005 [arXiv:1203.6139] [InSPIRE].

[40] G.A. Chirilli, L. Szymanowski and S. Wallon, Uncovering the triple Pomeron vertex from Wilson line formalism, Phys. Rev. D 83 (2011) 014020 [arXiv:1010.0285] [InSPIRE]. 
[41] A.H. Mueller, Small-x Behavior and Parton Saturation: A QCD Model, Nucl. Phys. B 335 (1990) 115 [inSPIRE].

[42] N.N. Nikolaev and B.G. Zakharov, Color transparency and scaling properties of nuclear shadowing in deep inelastic scattering, Z. Phys. C 49 (1991) 607 [InSPIRE].

[43] I.V. Anikin, D.Y. Ivanov, B. Pire, L. Szymanowski and S. Wallon, QCD factorization of exclusive processes beyond leading twist: $\gamma_{T}^{*} \rightarrow \rho_{T}$ impact factor with twist three accuracy, Nucl. Phys. B 828 (2010) 1 [arXiv:0909.4090] [InSPIRE].

[44] I.V. Anikin, D.Y. Ivanov, B. Pire, L. Szymanowski and S. Wallon, On the description of exclusive processes beyond the leading twist approximation, Phys. Lett. B 682 (2010) 413 [arXiv: 0903.4797] [INSPIRE].

[45] A. Besse, L. Szymanowski and S. Wallon, The Dipole Representation of Vector Meson Electroproduction Beyond Leading Twist, Nucl. Phys. B 867 (2013) 19 [arXiv:1204.2281] [INSPIRE].

[46] K.J. Golec-Biernat and M. Wusthoff, Saturation effects in deep inelastic scattering at low $Q^{2}$ and its implications on diffraction, Phys. Rev. D 59 (1998) 014017 [hep-ph/9807513] [INSPIRE].

[47] K.J. Golec-Biernat and M. Wusthoff, Saturation in diffractive deep inelastic scattering, Phys. Rev. D 60 (1999) 114023 [hep-ph/9903358] [INSPIRE].

[48] D.Y. Ivanov and M. Wusthoff, Hard diffractive photon-proton scattering at large $t$, Eur. Phys. J. C 8 (1999) 107 [hep-ph/9808455] [INSPIRE].

[49] G. Beuf, NLO corrections for the dipole factorization of DIS structure functions at low $x$, Phys. Rev. D 85 (2012) 034039 [arXiv:1112.4501] [INSPIRE].

[50] H1 collaboration, F.D. Aaron et al., Measurement of Dijet Production in Diffractive Deep-Inelastic Scattering with a Leading Proton at HERA, Eur. Phys. J. C 72 (2012) 1970 [arXiv:1111.0584] [INSPIRE].

[51] H1 collaboration, C. Adloff et al., Diffractive jet production in deep inelastic $e^{+} p$ collisions at HERA, Eur. Phys. J. C 20 (2001) 29 [hep-ex/0012051] [INSPIRE].

[52] ZEUS collaboration, S. Chekanov et al., Diffractive photoproduction of dijets in ep collisions at HERA, Eur. Phys. J. C 55 (2008) 177 [arXiv:0710.1498] [inSPIRE].

[53] H1 collaboration, F.D. Aaron et al., Diffractive Dijet Photoproduction in ep Collisions at HERA, Eur. Phys. J. C 70 (2010) 15 [arXiv:1006. 0946] [INSPIRE].

[54] M. Klasen and G. Kramer, Factorization breaking in diffractive dijet photoproduction, Eur. Phys. J. C 38 (2004) 93 [hep-ph/0408203] [inSPIRE].

[55] M. Klasen and G. Kramer, Review of factorization breaking in diffractive photoproduction of dijets, Mod. Phys. Lett. A 23 (2008) 1885 [arXiv:0806.2269] [INSPIRE].

[56] A.B. Kaidalov, V.A. Khoze, A.D. Martin and M.G. Ryskin, Unitarity effects in hard diffraction at HERA, Phys. Lett. B 567 (2003) 61 [hep-ph/0306134] [INSPIRE].

[57] H1 collaboration, A. Aktas et al., Diffractive open charm production in deep-inelastic scattering and photoproduction at HERA, Eur. Phys. J. C 50 (2007) 1 [hep-ex/0610076] [INSPIRE]. 